\title{
IMRP: Interference-aware Multicasting for Multiple Flows in WMNs
}

\author{
By: \\ Behzad Farmani \\ A thesis \\ Presented to Ryerson University \\ In partial fulfillment of the \\ Requirements for degree of \\ Master of Applied Science \\ In the program of \\ Computer Networks \\ Department of Computer Networks \\ Faculty of Electrical and Computer Engineering \\ Toronto, Ontario, Canada. 2015 \\ (C) Behzad Farmani
}




\section{Author's Declaration}

I hereby declare that I am the sole author of this thesis.

I authorize Ryerson University to lend this thesis or dissertation to other institutions or individuals for the purpose of scholarly research.

Author's signature:

I further authorize Ryerson University to reproduce this thesis or dissertation by photocopying or by other means, in total or in part, at the request of other institutions or individuals for the purpose of scholarly research.

Author's signature: 


\section{Abstract}

\section{Behzad Farmani, Interference-aware Multicast Routing for Multiple Flows in WMNs MASc. , Computer Networks, Ryerson University, 2015}

Multicast is an important routing service for many of fundamental applications such as Webcasting, Video Streaming or Conferencing, Online Games. On the other hand, as much as Wireless Mesh Networks (WMNs) are growing and become more complex, the need for modification of Multicast Routing for WMNs with different constraints are increased as well. Therefor Researches on multicasting in WMN is still in initial stage.

We focused on Multicasting over Single Channel WMNs with multiple group flows scenario. The main challenge on this scenario is the impact of interference between multiple flows on the performance of multicast flows. By increasing number of receiver nodes in a single channel area, the interference caused by multiple simultaneous flows in the network cannot be fully avoidable, but implementing a proper routing helps to improve the performance of the flows.

In this research, we develop a new interference-aware routing metrics and implement it in our purposed routing scheme (IMRP) for multicasting multiple flows in WMNs. Our purposed metrics takes some new defined attributes of routing path into account. The purposed scheme is evaluated by simulating on Riverbed Modeller 17.5. Simulation and testbed experiments show that our proposed routing significantly improves the total Throughput as well as total Packet Delivery Ratio of multicast flows compared to existing standards. 


\section{Acknowledgement}

I would like to express my sincere gratitude to Dr. Muhammad Jaseemuddin, my thesis supervisor for his patient supervision, and help leading towards this thesis.

Also I would like to express my thanks to Dr. Boby Ngok-Wah Ma and appreciate his continued support and guidance.

I am also thankful to Dr. Isaac Woungang, and Dr. Kamran Rahemifar for their time, guidance and advice.

Finally, my special thanks to my family, my parents for their great support and encouragement during my Master's studies. 


\section{Table of Contents}

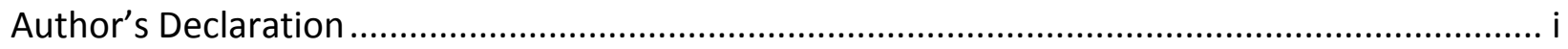

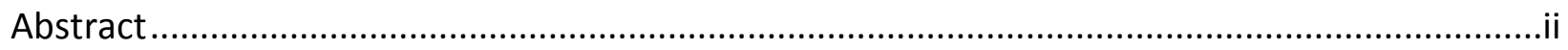

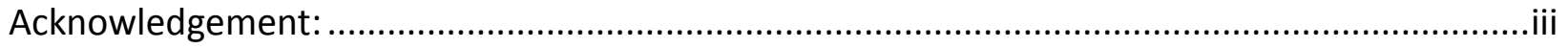

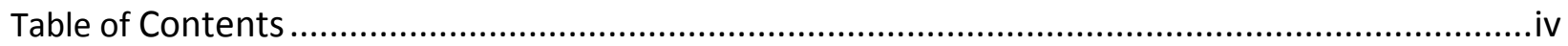

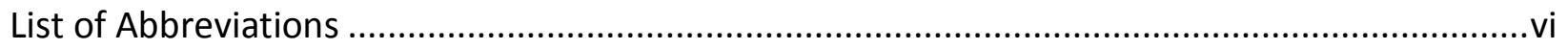

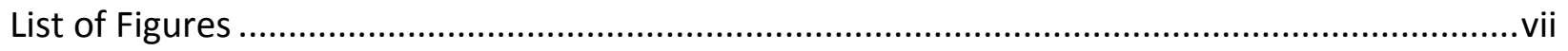

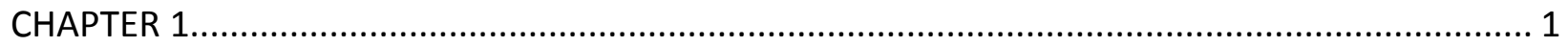

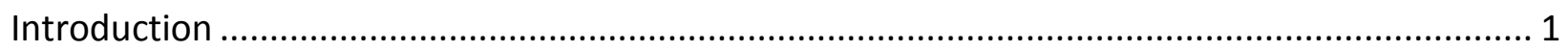

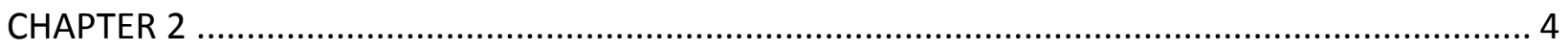

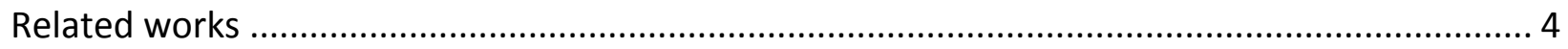

2.1 General Multicast Routing Protocols ................................................................. 4

2.2 Multicast Routing Protocols for Wireless Infrastructures ..................................... 6

2.2.1 MAODV, Multicast Ad hoc On-demand Distance Vector ..................................... 6

2.2.2 ODMRP, On-Demand Multicast Routing Protocol............................................... 7

2.2.3 ABAM, Associativity-Based Ad-hoc Multicast Routing ........................................ 8

2.2.4 ADMR, Adaptive Demand-Driven Multicast Routing ............................................. 8

2.2.5 MZRP, Multicast Zone Routing Protocol........................................................... 8

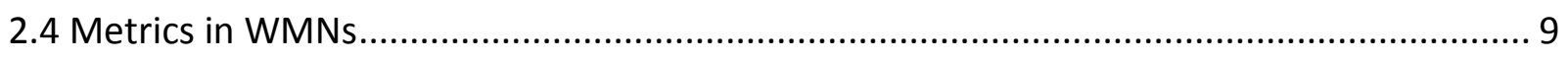

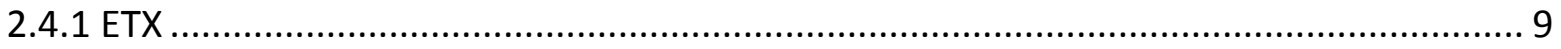

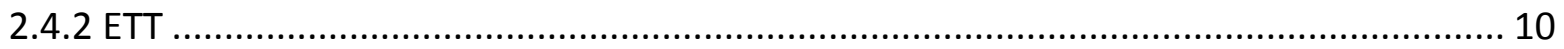

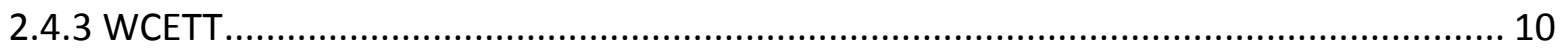

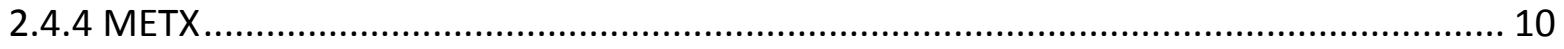




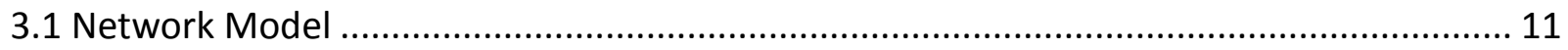

3.2 Wireless Transmission and Interference Model ...................................................... 12

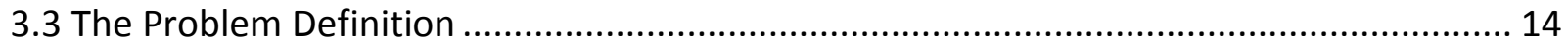

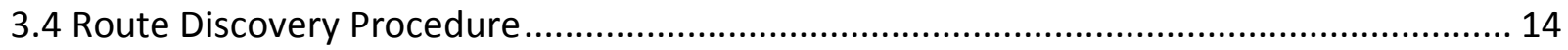

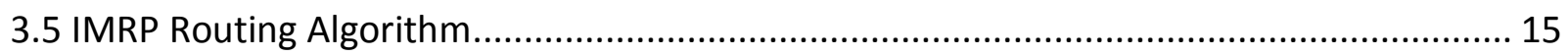

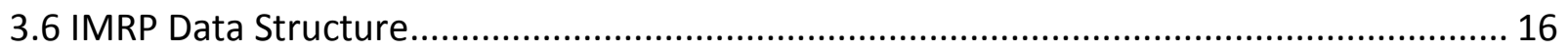

3.7 Paths Attributes and Metric Components ............................................................. 18

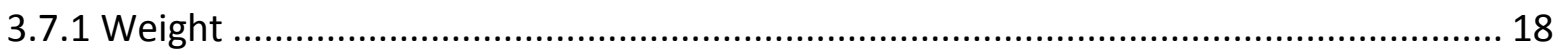

3.7.2 Local Interference Cost per node LIC (i ) ..................................................... 20

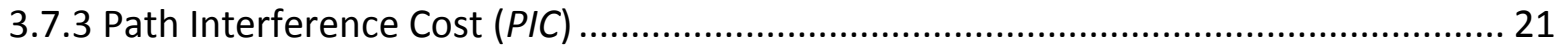

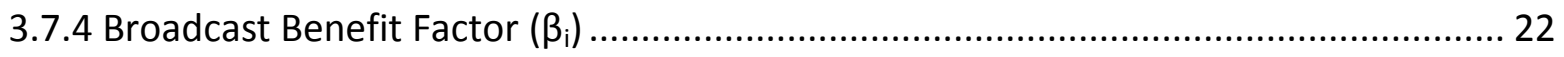

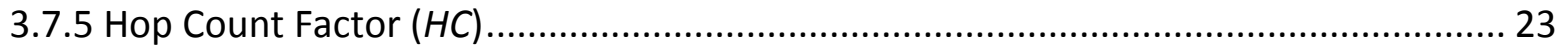

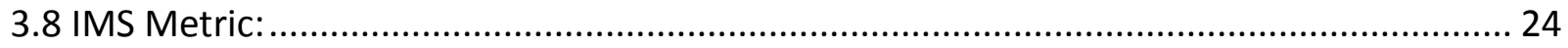

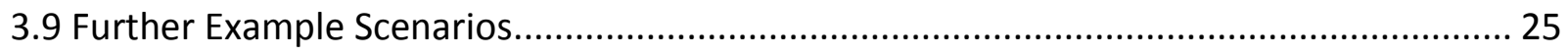

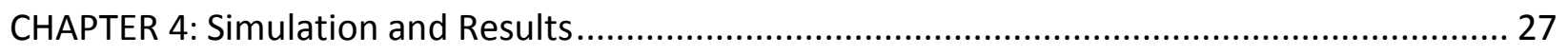

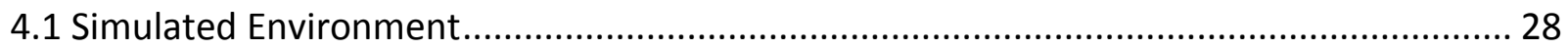

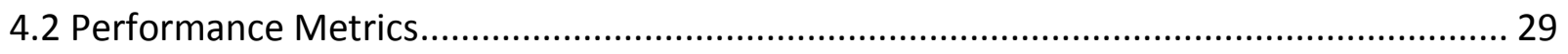

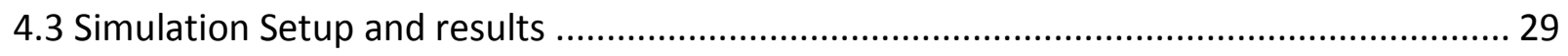

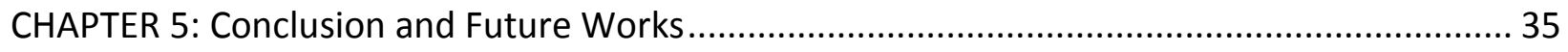

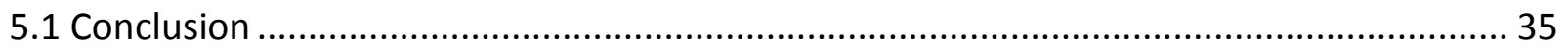

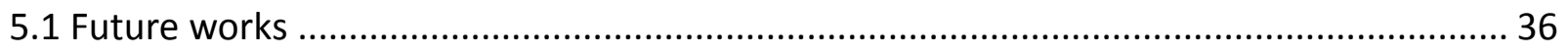

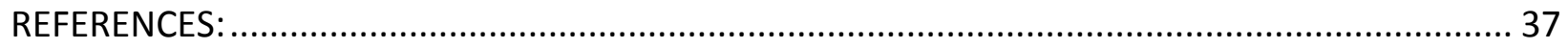




\section{List of Abbreviations}

\begin{tabular}{|c|c|}
\hline ANT & Adjacent Nodes Table \\
\hline AODV & Ad-hoc On-Demand Routing Protocol \\
\hline CBT & Core Based Tree \\
\hline ETX & Expected Transmission Time \\
\hline $\mathrm{HC}$ & Hop Count \\
\hline IGMP & Internet Group Management Protocol \\
\hline IMRP & Interference-Aware Multicast Routing Protocol \\
\hline IMS & Interference Aware Multicast Metric \\
\hline LIC & Local Interference Cost \\
\hline LSA & Link State Acknowledgment \\
\hline MCAST & Multicast Activation \\
\hline MOSPF & Multicast Extension to OSPF \\
\hline ODMRP & On-Demand Multicast Routing Protocol \\
\hline PDR & Packet Delivery Ratio \\
\hline PIC & Path Interference Cost \\
\hline PIM & Protocol Independent Multicast Routing \\
\hline $\mathrm{RP}$ & Rendezvous Point \\
\hline SPT & Shortest Path Tree \\
\hline WMN & Wireless Mesh Networks \\
\hline
\end{tabular}




\section{List of Figures}

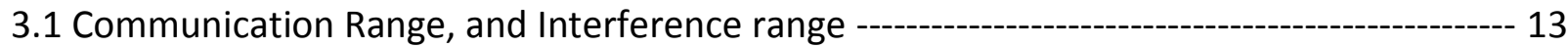

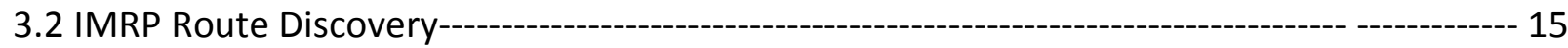

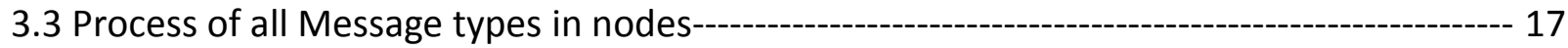

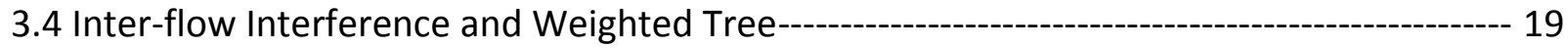

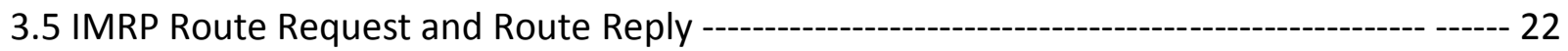

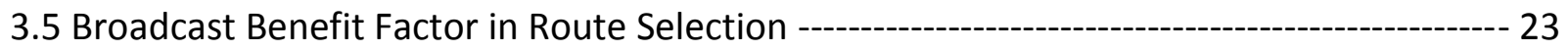

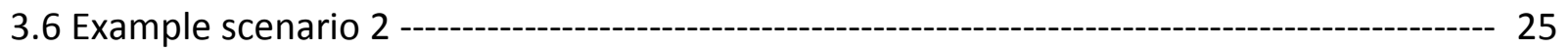

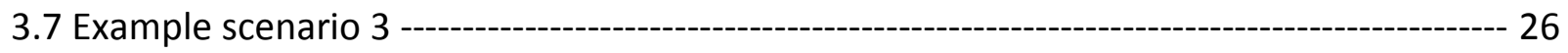

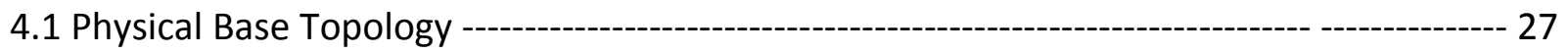

4.2 Average throughput over simulation for 6 and 12 receivers--------------------------------------- 30

4.3 Average throughput by increasing the number of receivers ---------------------------------- 32

4.4 Throughput analysis as function of multicast traffic load --------------------------------------- 33

4.5 Average PDR for both PIM-SM and IMRP with increasing receivers ----- --------------------- 34 


\section{Chapter 1}

\section{Introduction}

The need for efficient network support of one-to-many and many-to-many applications led to continuously improvement and development of multicasting protocols on different network infrastructures. When it comes to multi-hop wireless networks the major concern is interference between multiple flows carrying multicast traffic in the nearby wireless links.

In Wireless mesh networks, equipped with omnidirectional antennas, the transmission channel is indeed a broadcast medium. Transmissions among neighboring nodes may interfere with each other because of contention for the shared wireless channel. One of the main problems for such networks is the reduction in total capacity due to interference between multiple simultaneous transmissions. When we implement multicasting especially for media streams, the continuous flows in direct neighbor nodes or adjacent nodes in Interference range of the receiver nodes, highlights the mentioned issue. Therefore, when it comes to multi-hop wireless mesh networks the major concern is interference among multiple flows carrying multicast traffic in the nearby wireless links.

In this research, we propose an interference-aware multicast routing scheme in a single-radio Wireless Mesh Networks for multiple flows. We focus on a specialized multicast routing over WMNs with stationary nodes, such as community wireless networks. We present a new metrics for multicast routing and incorporated our routing metric which is called Interference Aware Routing Metrics (IMS) in the proposed route discovery and path selection method which we call it IMRP. IMRP reduce the interference in the whole WMN network carrying multiple flows which belong to various multicast groups and, thereby improves network throughput. Broadcasting is one of the fundamental operations in Wireless Mesh Networks. The benefit point of broadcasting in multicast is delivering multicast packet from single node to all other 
neighbor nodes. However, broadcasting is also facing some issues like collision, severe contention, and congestion. Generally, in wireless networks 802.11 based CSMA/CA avoids interference and collision by conservative scheduling of transmissions. By using Carrier Sensing method, nodes before transmitting listen to the shared medium, and attempt to avoid collisions by transmitting only when the channel is sensed to be idle. It has been proven that CSMA/CA gives an efficient support for broadcasting and solves the hidden terminal problem. In 802.11, MAC layer unicast involves may optionally use RTS/CTS exchange before sending a data packet. Although, the RTS/CTS exchange avoids the hidden terminal problem, 802.11 MAC layer broadcast does not involve any RTS/CTS exchange before sending a broadcast packet. In case of having multicast in mesh networks, when a node send RTS, the CTS packets sent by multicast members may have high probability colliding, therefore in our proposed model we rely on CSMA/CA only without using RTS/CTS method.

On the other hand, in order to minimize the interference, we propose an interference metric for our routing algorithm. Since, minimum hop count is not appropriate metric for multicast routing, there are many research recently proposing different metrics by taking account of link quality in account. METX [33], ETX [23], ETT \& WCETT [24], and iAWARE [26] are some examples of metrics designed for multicast. In chapter 2 we review the mentioned metrics and analyze the advantages and disadvantages of them. In our proposed metric, we consider multiple attributes on links and path, one of them - Interference Cost- explicitly take the interference criteria in account, the others implicitly cause reducing the interference between multiple flows overall in the WMN networks.

The goal of our metric is to choose a join path to multicast Tree for a new receiver node with lower inter-flow interference, and highest throughput. Our metric ISM, implements some important attributes assigned to individual nodes running IMRP routing protocol and helps to construct the higher performance Trees for multiple groups in the entire WMN network.

We studied the performance of our metric by implementing it in a wireless testbed consisting of 50 stationary nodes, each equipped with 802.11 wireless cards. We find that in a single-radio 
environment with stationary nodes, our scheme significantly outperforms existing routing protocols.

\section{Thesis Outline}

This research is organized as follows: Chapter 2 reviews the general multicast routing first, and then the various multicast routing protocols proposed to Wireless networks including Multicast Ad hoc On-demand Distance Vector (MAODV) [14], ODMRP On-Demand Multicast Routing Protocol (ODMRP) [17], ABAM, Associativity-Based Ad-hoc Multicast Routing [20], ADMR, Adaptive Demand-Driven Multicast Routing [18] and MZRP, Multicast Zone Routing Protocol [19] are analyzed. We also review various metrics reported in recent researches and literatures. The most common and recent metric such as ETT, ETX, METX, and WECTT are reviewed in detail. In chapter 3, we introduce our network model and the new interference-aware multicast routing IMRP and route recovery procedure. Then we present the design of our new interference-aware metric called IMS and provide the various topology examples showing how each of metric component helps to reduce the overall interference and increase the throughput of multiple flows in the entire of the networks. 


\section{Chapter 2}

\section{Related works}

\subsection{General Multicast Routing Protocols}

Prior to discuss about multicast related researches on Wireless Mesh Networks, we quickly review the general protocols and techniques used for Multicasting in wired networks. Multicast routing has been extensively studied in wired networks. There are many proposed protocols for multicasting over wired networks for instance: Distance Vector Multicast Routing Protocol (DVMRP) [11], Multicast Extensions to Open Shortest Path First (MOSPF )[12], Core-Based Tree ( CBT), and Protocol Independent Multicast ( PIM) [13] .

DVMRP was the first multicast protocol which is tree-based and built on the principles of RIP unicast routing protocol and uses Hop count as it's metric. DVMRP has a built-in unicast routing protocol to facilitate finding source sub-networks relative to any router, and to help determine which interfaces were children with respect to any source. DVMRP implements RPM (Reversepath multicasting) algorithm to generate the multicast tree. RPM is an implicit-join algorithm which means by assuming the presence of downstream group members, the first datagram for any (source, group) pair is broadcast across the entire internetwork and then unused edges are pruned. Therefore it is also called "Broadcast-and-Prune".

MOSPF, Multicast Extension to OSPF are defined in RFC-1584 [12]. MOSPF routers maintain a current image of the network topology through the unicast OSPF link-state routing protocol. The elected DR MOSPF router from any sub-network, floods Group-Membership LSAs to all other routers in the backbone area. The MOSPF link-state database is simply the standard OSPF link-state database augmented by Group-Membership LSAs. Based on network-LSAs in the OSPF link-state database, a source-based shortest path tree is constructed using Dijkstra's algorithm. MOSPF's tree-building process is data-driven [15]. Although there is Group- 
Membership LSAs present in each area's link-state database, Multicast Trees are not built unless data is seen from source to a group. On the other hand, if the Link-state Database indicates no Group-Membership LSAs for the group, no tree will be built because there is clearly no group members present in that area. Because of this feature, MOSPF is an explicit-join protocol not Broadcast-and-prune protocol, despite being data-driven.

Protocol Independent Multicast (PIM) is indeed two protocols: PIM-Dense Mode and PIMSparse Mode. PIM-DM is based on reveres-path multicasting like DVMRP. But the one of the differences between those two is DVMRP has built-in routing protocol and PIMs can use any underlying routing protocol. The other difference is that PIM-DM is simply forwards multicast traffic on all non-incoming interfaces until explicit prune messages are received, unlike the DVMRP, which calculates a set of child interfaces for each source group pair. The default forwarding action of implicit-join routing protocols such as PIM-DM is to broadcast all traffic away from the source, while the default action of a sparse-mode protocol is to send traffic only where it has been explicitly requested.

PIM-SM is sparse-mode and designed to limit multicast traffic. So it builds shared trees which must be explicitly joined by downstream routers. When joining a group, each receiver uses IGMP to notify its directly attached routers, which in turn joins the multicast delivery tree by sending an explicit PIM-Join message hop by hop toward the group's RP. The source's PIM-SM router knows how to reach the RP and forward traffic from to RP. PIM-SM evolved from the core-based trees (CBT) approach, in that it employs the concept of a core (or Rendezvous Point in PIM-SM terminology) where receivers meet sources. PIM-SM can use either source-based (SPT-Shortest Path Tree) or shared Tree (RP-Tree). Once interested receivers have used IGMP to join a group, the sub-network's PIM-SM DR then issues PIM-Join messages on their behalf toward the group's RP. These join messages establish forwarding state in the intermediate routers which is cached to support future forwarding decisions. If the rendezvous point receives a packet for which there is no pre-established forwarding state, the packet is dropped.

PIM-DM, DVMRP, and MOSPF are not well suitable for multicast topologies in which group members and senders are sparsely distributed. PIM-DM, DVMRP, and MOSPF do not provide 
the most efficient multicast delivery service, in the sense that they use more bandwidth or resources than might be necessary in a protocol designed for the sparse case. These protocols deliver packets efficiently once they have built their tree, but the tree building process is inefficient. The DVMRP periodically broadcasts multicast data packets over many links that do not lead to group members.

CBT, Core-Based Trees is a multicast architecture that is based on a shared delivery tree. Similar to PIM-SM, CBT is protocol independent. CBT employs the information contained in the unicast routing table to build its shared delivery tree. It does not care about how the unicast routing table is derived, only that a unicast routing table is present. This feature makes CBT to be a protocol independent, meaning there is no requirement of any specific unicast routing protocol.

However, when it comes to wireless networks there are additional specific objective functions need to be considered such as bandwidth limitation, energy consumptions, and interference issues. According to the concepts, and techniques used in our research, in this chapter we briefly introduce the previous works related to Interference in Wireless Networks, Routing Metric design and Multicasting protocols and schemes for wireless mesh networks.

\subsection{Multicast Routing Protocols for Wireless Infrastructures}

In this section we review some of the existing and primary multicast routing protocols designed on deferent Wireless networks infrastructures.

\subsubsection{MAODV, Multicast Ad hoc On-demand Distance Vector}

MAODV is a tree-based multicast protocol [14] which is an extension of Ad hoc On-Demand Distance Vector (AODV) routing protocol, and there are some similarities with AODV [18] such as packet formats, configuration parameters. The same packet type and formats for "Route Request" and "Route Reply" used by AODV have been implemented in MAODV as well. Route Requests (RREQs), Route Replies (RREPs), Multicast Activations (MACTs), and Group Hellos 
(GRPHs) are the message types utilized by the multicast operation AODV. Based on the method of Route Discovery in MAODV, when a node need to join a multicast group or it receives a multicast traffic for a group but there is no route to that group, it issues a route request (RREQ) message. Only the members of the multicast group tree respond to join RREQ. If an intermediate node receives a join RREQ for a multicast group of which it is not member or it receives a route RREQ and it does not have a route to that group. It rebroadcast the RREQ to its neighbors. The objective designs in this protocol are node mobility and reducing the energy consumption.

\subsubsection{ODMRP, On-Demand Multicast Routing Protocol}

ODMRP has target the ad-hoc wireless networks with limited power and bandwidth [17], and also mobility of the hosts. Therefore it applies on-demand route construction for multicast traffic, and membership maintenance. ODMRP is a source-initiated and mesh-based protocol and uses a group of nodes as forwarder or relaying nodes to lead data to downstream nodes, based on shortest-path in between.

When a source has a multicast packet to send, but doesn't have any route to the multicast group, it periodically floods the Join-REQUEST packet to the entire network. When an intermediate node receives a packet containing Join-Request, and detects that the packet is not duplicated it records the upstream node ID, and rebroadcast it. When a multicast receiver node receives the Join-REQUEST, it forms or update Member Table by new Source entry. Join-Tables are broadcasted periodically to the neighbors. When the neighbor nodes receive the Table, they check if the next node ID in any of entries is their own ID, then adjust its downstream port for a multicast group, sets FG flag, and then broadcast its own Join Table. This process continues in the entire network until reach to multicast Source through the shortest path. The feature of providing redundant paths in mesh form multicast topology in ODMRP, makes it suitable for dynamic environment and robustness for receivers' motility. In contrast, tree-based 
protocols provides only one path between sender and receiver, and it has a higher probability of collision.

\subsubsection{ABAM, Associativity-Based Ad-hoc Multicast Routing}

Associativity-Based Ad hoc Multicast is another On-Demand multicast routing protocol proposed by Guillermo, and Santithorn [20]. The tree construction is source-based, and unlike ODMRP doesn't use periodic flooding but flooding based on Join-Request. The key idea in ABAM is association stability concept which was introduced in Forwarding Group Multicast Protocol [21]. The authors showed lower end-to-end delay in comparison with ODMRP. Although ABAM has also intended to wireless infrastructure with mobile nodes, it has lower throughput while increasing the node mobility.

\subsubsection{ADMR, Adaptive Demand-Driven Multicast Routing}

ADMR is source-based tree routing [18]. When there are many sources in multicast group, separate multicast trees are constructed for each of the sources. When a new node need to join a multicast group, it flood a Request Packet to the entire network, and after replying the requests by sources, the receiver node send a Join-packet to the answering source. For each source an individual tree has been maintained in network.

\subsubsection{MZRP, Multicast Zone Routing Protocol}

MZRP also constructs source based trees like ADMR. It is source-initiated protocol. Each node keeps the topology information of its zone [19]. MZR performs zonal routing; hence, the flooding of control packets is less expensive. Compared to approaches based on shared trees, the use of source-based trees creates much more state at routers participating in many groups, 
each with multiple sources. The drawback of MZRP is the longer required time for a new receiver to join the source, specially, when the receiver is too far from the source.

\subsection{Metrics in WMNs}

The most basic routing metric is Hop Count which has been used in many distance vector routing protocols such as AODV ( Ad-hoc On-demand Distance Vector ) [11] , DSR ( Dynamic Source Routing) [22]. All proposed protocols in [27], [B28], [29], [30], [31], [32], and [33] have used the hop count as metric. However, minimum hop count is not an appropriate criteria for route selection in Wireless Mesh Networks in the presence of many other factors like interference, congestion, delay, etc. In order to consider the other influencing factors like congestion control, scalability, and to select a path with higher throughput, there have been many researches proposing some new metrics. In this part we review some of the previous works and proposed metrics in WMN networks:

\subsubsection{ETX}

Expected Transmission Count (ETX) proposed by Douglas et .al in [23] is a metric based on measuring link loss characteristics. ETX of link is calculated by using forward and reverse delivery ratios of some dedicated probe packets transmitted along the link:

$$
E T X=\frac{1}{\mathrm{df}+\mathrm{dr}}
$$

Where $d_{f}$ and $d_{r}$ are Forward Delivery Ratio, and Reverse Delivery Ratio respectively.

ETX of a route or path, accordingly, is sum of the ETX in each link in the path between source and destination. Disadvantages of ETX are: 1- Loss ratio may vary for different packet size 2- it doesn't take into the account for link bandwidth and intra-flow interference. 


\subsubsection{ETT}

ETT is an improved metric over ETX, by taking the link bandwidth into account [24]. By definition of:

$$
\mathrm{ETT}=\mathrm{ETX} *(\text { Packet Size / Bandwidth })
$$

ETT is in fact the time a data packet need to be successfully transmitted to each neighbor. ETT can adjust ETX for various packet size and various link bandwidth. However, the disadvantage of ETT is the existing load of the link is not considered.

\subsubsection{WCETT}

In [24] Draves, et .al had defined the Weighted Cumulative ETT by considering channel diversity on each node, to cover the multi-radio multi-hop networks and the value of WCETT is expressed as

$$
\text { WCETT }=(1-\beta) * \sum_{k=1}^{n}\left(E_{T T}\right)+\left(\beta * \max _{1<j<k} X_{j}\right)
$$

Therefore a weight is assigned to each link based on Expected Transmission Time (ETT). Although WCETT explicitly considers the interference among links that use the same channel, but as per experimental results in [26], it can't capture the inter-flow interference when there are multiple flows in the network.

\subsubsection{METX}

METX was designed as an extension of ETX for multicast environments [33]. The value of METX estimates number of transmissions needed to deliver a multicast packet to all of the joined group members. The difference between METX and ETX is that METX qualifies the link quality in bit level whereas ETX measures link quality in packet level. Similar to ETX, and WCETT, METX is also a probing base metric. The advantage of this metric comparing to other two is improvement on throughput and reliability and minimum energy consumption. However, to the best of our knowledge none of the metrics provided on recent research such as [1] to [8] consider the overall throughput of the multiple flows simultaneously in the network. 


\section{Chapter 3}

\section{Interference-aware Multicast Routing (IMRP)}

In this chapter, we first present our network model that we used to design a multicast routing protocol. Then, we describe our composite metric with detail discussion on each component separately, followed by metric formula. Finally, we present our proposed Interference-aware Multicast Routing (IMR) algorithm for routing multicast traffic within a wireless mesh network.

\subsection{Network Model}

We assume a Wireless Mesh Network (WMN) consist of wireless mesh routers, which form the mesh infrastructure. We assume. The first channel in all nodes is used for unicast traffic only and the second channels are used for multicast flows. It employs IEEE 802.11 MAC for the unicast channel. We propose an interference-aware composite metric (IMS) for multicast route selection that avoids both inter-flow and intra-flow interferences. We also design a route discovery algorithm that maintains multiple multicast trees to support multiple groups and flows. In our model, each mesh node has two wireless channels. The other assumptions and model specifications in our study are as follows:

- Mesh nodes in WMN network are stationary nodes.

- The thermal noise level is lower than the Interference level and negligible

- The multicast routing is based on PIM-SM model

- Mesh nodes get their location information by GPS

- Multicast sources are located outside the WMN and the multicast traffic from the sources enter the wireless network from multiple Gateways

- All of the nodes are equipped with Omni-directional antennas running 802.11 MAC Layer.

- Transmit power is the same for all of the nodes in the wireless network. 
- The environmental condition is the same for all the nodes, which results in the same path gain/loss.

\subsection{Wireless Transmission and Interference Model}

In order to qualify the transmission links between any two nodes in the Multicast Tree in the presence of interference, we considered two different interference models which have been used in the literatures: physical and protocol model.

\subsubsection{Physical interference model:}

In physical interference model, a message can be transmitted successfully between two nodes if the received signal to interference plus noise ratio (SINR) exceeds a given threshold $\gamma_{\text {thr }}$ that is,

$$
S I N R=\frac{\operatorname{Pr}, \mathrm{i}}{\sigma_{n}^{2}+\sum_{j \neq i} \operatorname{Pr}, \mathrm{j}}>\gamma_{\mathrm{thr}}
$$

Where $\gamma_{\text {thr }}$ is the minimum SINR required for a successful message reception. $\sigma_{n}^{2}$ is the ambient noise power, $\operatorname{Pr}, i$ is the power received from the source $i$, and $\operatorname{Pr}, j,(j \neq i)$ is the power received from a set of transmitters that are transmitting simultaneously with the source which can cause interference.

The physical interference model imposes realistic condition for successful reception and it is widely considered as a reference model for physical layer.

\subsubsection{Protocol Interference model}

In this model, any two nodes can communicate and their transmission is successful if and only if the receiving node is located within the transmission range of the intended transmitting node and is outside the interference range of any other node that is actively transmitting on the same band. 


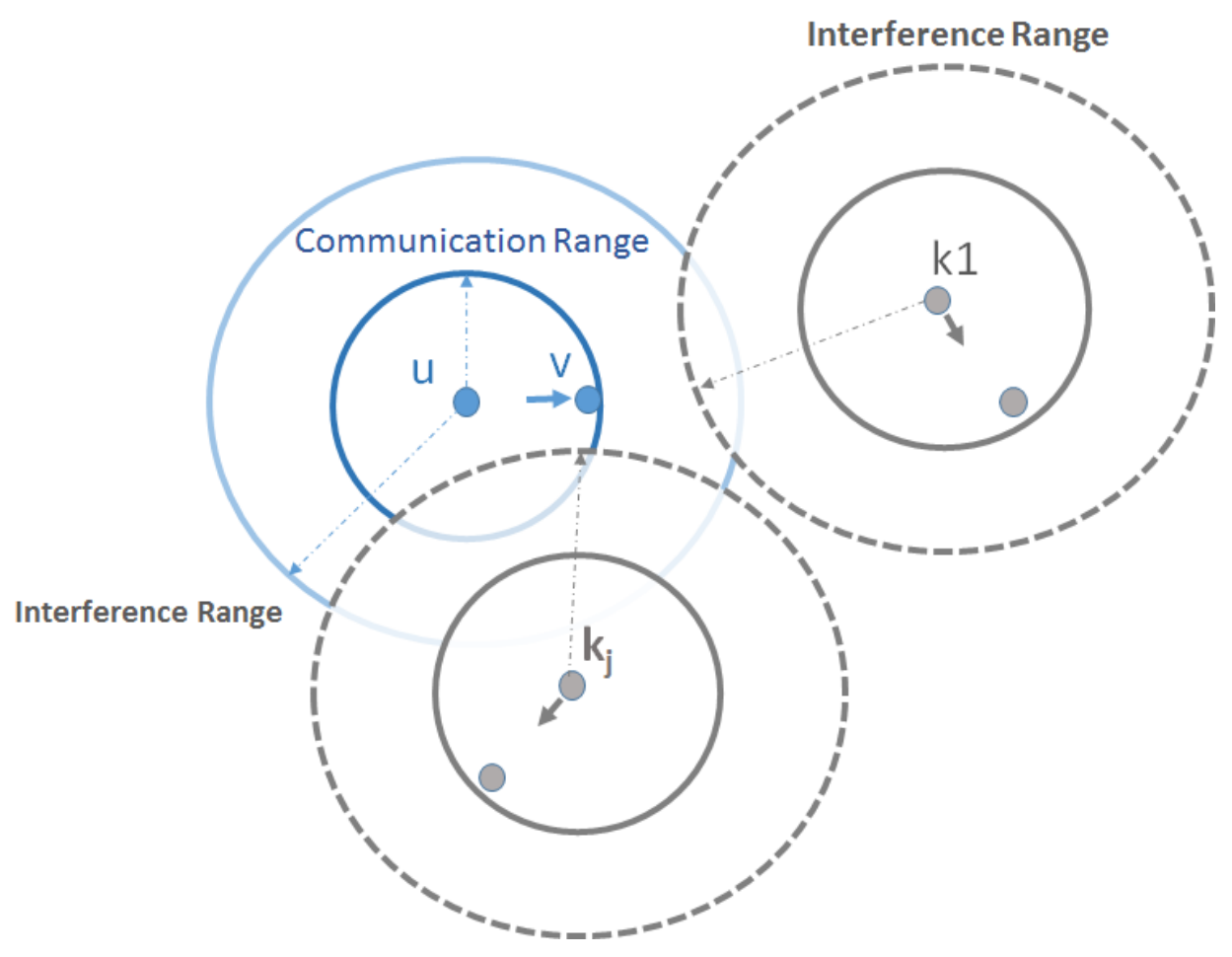

Figure 3-1: Communication range, and Interference range

In the protocol interference model, the transmission from node $\mathbf{u}$ to node $\mathbf{v}$ in Figure $3-1$ is successful if both of the following conditions are satisfied for every other node $\mathrm{k}_{\mathrm{j}}$ that is simultaneously transmitting or receiving, that is:

1. $v \in \mathrm{N}_{\mathrm{T}}(\mathrm{u}) \Rightarrow \mathrm{d}(\mathrm{u}, \mathrm{v})<\mathrm{R}_{\mathrm{T}}$

2. $\vartheta \mathrm{k}_{\mathrm{j}} \mid \mathrm{kj}$ is transmitting $\Rightarrow \mathrm{d}\left(\mathrm{k}_{\mathrm{j}}, \mathrm{v}\right)>\mathrm{R}_{\mathrm{I}}$

\section{Where:}

$\mathrm{N}_{\mathrm{T}}(\mathrm{u})$ is set of direct neighbour nodes located within Communication Range of node $\mathrm{u}$.

$\mathrm{R}_{\mathrm{T}}$ is Transmission Range (Communication Range) of the node. $\mathrm{R}_{\mathrm{I}}$ is Interference Range of transmitting node. $d(u, v)$ as the Euclidean distance between nodes $u$, and $v$. 
We assume uniform transmission range $R_{T}$ and Interference range $R_{l}\left(>R_{T}\right)$ for all the nodes in WMN, as shown in Figure 3.1. We assume 802.11 CSMA/CA as medium access control scheme. Therefore the all other neighbor nodes $\mathrm{N}_{\mathrm{T}}(\mathrm{u})$ within transmission range of node $\mathrm{u}$ which are sensing the transmission carrier of $u$, will be prevented of sending multicast streams. For any given Mesh Router we can also define the set of adjacent nodes which are located within interference range of node $u$ : NIF $(u)=\left\{x \mid d(u, x)<R_{I}\right\}$

NIF(u) represents all nodes within the interference range of node $u$.

\subsection{The Problem Definition}

The maximum data rate that can be achieved in a multicast tree is limited by the data rate of the bottleneck link. The data rate of the bottleneck links is determined by the amount of interference experienced by the receiver of a link.

\subsection{Route Discovery Procedure}

We propose a multicast route discovery procedure to create multiple multicast trees in which the forwarder nodes within the trees use link-layer broadcast. We create the receiver initiated, and source-based tree creation. We also define a composite metric used in our algorithm that is based on interference cost and broadcast benefit of a link.

In order to describe the algorithm and composite metric we introduce some elements used in our metric. In the following section we define each element separately with simplified examples showing the role and effect of each components of the metric, then provide the algorithm for collecting the required information from mesh nodes, processing, calculating the metric and distributing them within the network.

1- Path Interference Cost (PIC): A comparable value for indicating the impact of interference caused by any selective path on all flows receive by all group nodes.

2- Broadcast Benefit $\left(\beta_{\mathrm{i}}\right)$ : Number of receiver nodes around a multicast member $\mathrm{i}$ which are served by taking the benefit of link-layer broadcast.

3- Hop Count to nearest Tree Member ( HC) 
4- Tree's Weight : An attribute of any Tree node showing how many receiver nodes have joined in downstream.

\subsection{IMRP Routing Algorithm}

Our proposed IMRP algorithm builds receive-initiated multicast tree. We assume one root node is configured per group that receives multicast traffic from one of the Gateways of WMN. By running IMRP on any node regardless of joining to any group, some tables are formed and initialized. The database structures will be described further in section 3.3.2. Similar to ODMRP and MAODV, there are 3 steps route discovery process in our algorithm. In IMRP the tree building process starts with receiver unlike ODMRP that starts by flooding from Sender/Source. Hence IMRP is basically a receiver-initiated method. Typically, the tree building process started by a receiver goes through three phases that are described below.

\section{Phase 1: ROUTE-REQUEST}

When a node wants to join a group, it broadcasts a ROUTE-REQUEST to the neighbors as shown in Figure 3-2. If a neighbor either has already joined the tree (group, source) or has the potentially best route to the requested tree (group, source), it goes to the next phase, otherwise it rebroadcasts the request.

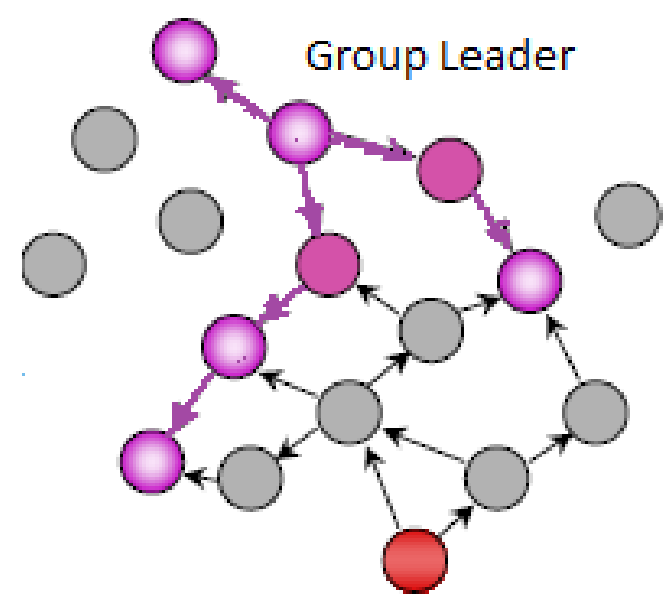

New Receiver

Figure 3-2 IMRP Route Discovery 


\section{Phase 2: ROUTE_REPLY}

Once any node matches the requested (group, source) with its available best route in IMR Table, it adds a potential downstream member in its IMR Table and puts its best route's attributes in ROUTE_REPLY packet and replies it. Along the reply path, any intermediate node that receives multiple replies from its neighbors, calculates the final metric, chooses the best potential upstream node, and then sends the ROUTE_REPLY back to the preceding node. Once we introduced all metric components in detail in section 3.4.3, we will describe the processing of ROUTE-REPLY packets in detail and the algorithm of distributing the best route attributes in ROUTE-REPLY.

\section{Phase 3: JOIN-CONFIRM}

The third and final step is establishing the new tree leafs from the requesting receiver up to the best tree member according to the best reverse path. After calculating the metric for multiple offered routes from neighbors, the node selects one route and sends back the Join-Confirm packet accordingly to intermediate nodes. It also changes the route Flag status from "Potential" to "Active".

\subsection{IMRP Data Structures}

Network nodes running IMRP are required to maintain the following data structures.

Adjacent Nodes Table ( ANT Table): Each node stores the location information of any nodes within its interference area. When any node joins a multicast group it broadcasts the Hello Packet containing its location and adjacent nods' info. Once the neighbour nodes recive the Hello Packets, they updates their ATN table and check if any node's location is within their interferece area, they add the new entry in their ANT tables and then broadcsts the new Hello packet.

IMR Table:

All information received by RouteReply Packet from upstream nodes are stored in IMR table. Initially any path offered by upstream nodes are tagged with "Potential Status' by changing the join flag to "Potential". The Join flag conatins two status: 1-Potential 2-Active. The IMR Join 
table includes all Metric componenets like Path Cost, Broadcast Benefit $\left(\beta_{i}\right)$, Weight $(W), Q$, and upstream node ID, and Route Reply Sequence.

In figure 3-3 we show the required actions by nodes upon receiving any type of the above messages:

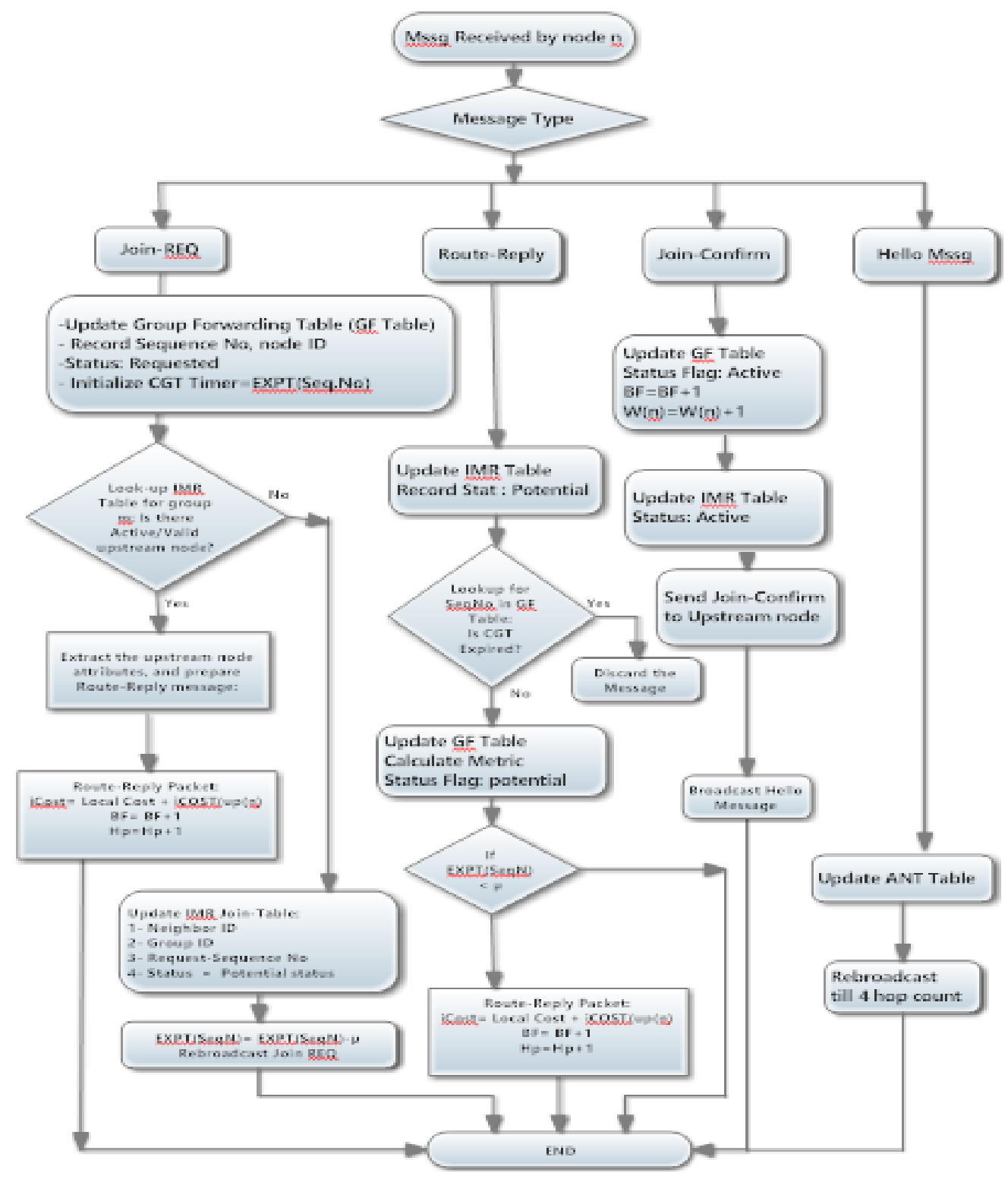

Figure 3-3 Process of all Message types in nodes 


\subsection{Paths Attributes and Metric Components}

In this section we discuss different attributes used in defining the metric. We also discuss how they are distributed within the network. Before we introduce our Interference Cost, we define a new attribute called "Weight" which is incorporated in our purposed Interference Cost.

\subsubsection{Weight}

Since our focus is on allowing multiple flows dedicated to multicast traffic in WMN, evaluating the effect of inter-flow interference on both interfering flows at the same time may help us significantly reduce the impact of interference on overall throughput of the flows on the entire WMN. In order to do this, we assign a weight attribute to the member nodes in the multicast tree, so that the value of the weight for any node in the tree indicates the number of receivers connected in downstream. Therefore, the nodes close to the root of the tree have higher weight than the leaf nodes. Assume node $A$ in figure 3.3 needs to join multicast group 3 . There are two paths available for $\mathrm{A}$, the one path via node $\mathrm{N}$ and another path via node $\mathrm{M}$ which are named path $a$, and path $b$, respectively as shown in Figure 3-3. Path a will interfere with flow $\mathrm{G} 1$, and path $b$ will interfere with flow $\mathrm{G} 2$. We can distinguish between the two paths $a$ and $b$ in terms of the impact of interference on total throughput of groups G1, and G2 which will be suffered by interference experienced by $\mathrm{N}$ and $\mathrm{M}$. Since node $\mathrm{N}$ mutually interferes with node $\mathrm{H}$ by weight 6 and node $M$ interfere with node $F$ by weight 2, we can associate higher interference cost of path a than path $b$. 


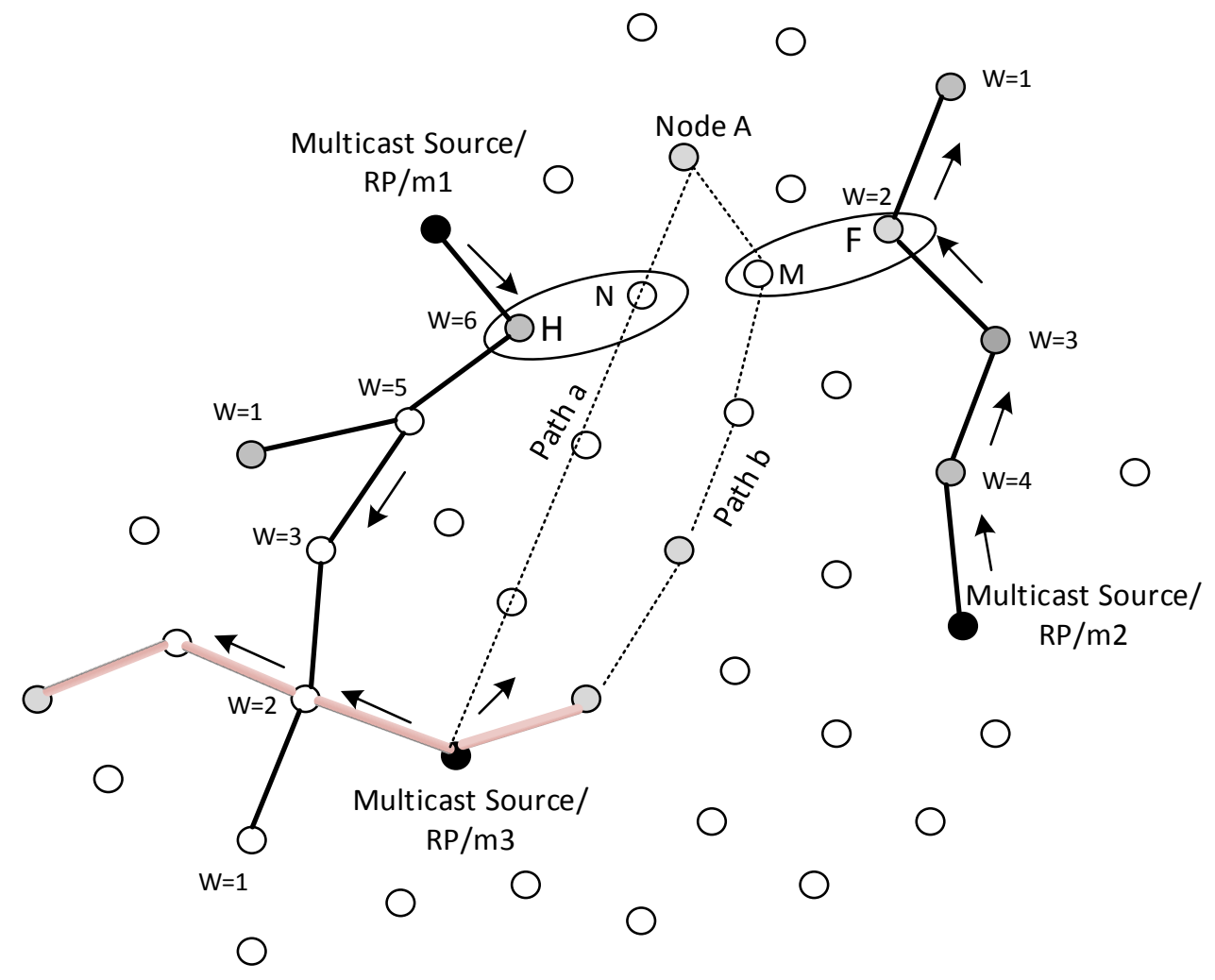

Figure 3-4: Inter-flow Interference and Weighted Tree

For any node $\mathrm{K}$, we have

$W_{m}(\mathrm{k})=$ Number of nodes downstream of $\mathrm{k}$ that have joined the group $\mathrm{m}$.

One of the advantages of using this attribute is preventing too many reconfigurations of the multicast tree. When the number of groups (flows) are increased in WMN, frequent topology changes may occur in the tree because of inter-flow interference, this may cause instability of multicast routes and reduces the total throughput. Therefore, by using weight attribute in our metrics which evaluates and takes the impact of new flows over existing flows' performance into account, we are able to reduce the reconfiguration and changes in tree constructions while running new flows in the network.

We incorporate this attribute in the Interference Cost of our composite metric that is described in section 3.5. 


\subsubsection{Local Interference Cost per node $L I C(i)$ :}

We assume the interference at a node linearly varies with its distance from the interfering node. In the other words, as much as interfering nodes get close to the receiver nodes, the interferer signal power is increased and probability of bit error is increased. By this simplification we can estimate the degradation of delivery ratio in specific node.

Hence, we define the local interference cost as:

$$
L I C(i)=\sum_{k \in N I F(i)}\left\{\frac{R_{I}-d(i, k)}{R_{I}} * \sum_{m}\left[Q_{m}(k) * W_{m}(k)\right]\right\} \mid \mathrm{k} \neq \mathrm{i} \& \mathrm{k} \in \mathrm{NIF}(\mathrm{i})
$$

Where:

Where: $R_{I}$ is interference range, $\mathrm{d}(\mathrm{i}, \mathrm{k})$ is distance between node $\mathrm{i}$ and interfering node $\mathrm{k}$

NIF(i) is the set of adjacent nodes which were recorded in ANT table of node i

$Q$ factor is defined as:

$Q_{m}(k)= \begin{cases}0 & \text { if Node } K \text { has not joined to any multicast group } \\ 1 & \text { if Node } K \text { is forwarding node for multicast group } m\end{cases}$

So that the maximum distance of interfere node within interference range of transmitter will result minimum value of interference factor which we define it below by $(i)$. Since we also consider the increasing cost of interference to adjacent flows in our metric, and according to the Tree Weight concept explained in previous section, we simply multiply it with the weight factor of the interferer node as well as $Q$ factor indicating the number of groups node $k$ joined. The reason of assuming comparable increasing cost for adjacent nodes is that when we take LIC $(i)$ as interference factor, we take this value as a Local Interference Cost for receiver node $\mathrm{i}$. Obviously, local interference cost is not accounted when node i calculate the best metric to join to one of upstream nodes as best route, because the value will be equally added to all potential upstream paths metric. Once node ' $i$ ' is already joined to one upstream node, in case of receiving join request from downstream nodes, it will add local interference cost to the path interference cost and advertise it as its best route attributes. In this case, node i potentially 
becomes a transmitter node and will influence on loss rate of adjacent interferer nodes which also play a receiver node from their own upstream nodes. As result our Interference cost factor indicates a comparable affecting cost for both interacted flows in overall.

\subsubsection{Path Interference Cost (PIC):}

We define Path Interference Cost at a receiver as sum of Local Interference Costs of all intermediate nodes from Group Leader node to the receiver node.

$P I C_{\text {path B }}=\sum_{\text {he path B }}\left(\sum_{\mathrm{k} \in \operatorname{NIF(h)}}\left(\frac{R_{I}-d(h, k)}{R_{I}} * Q_{m}(k) * W_{m}(k)\right)\right)$

When a node receives ROUTE-REQUEST message, the ROUTE_REPLY packet will include the Path Interference Cost from Group leader up to requested node. Assume node $\mathrm{j}$ sends a JoinRequest to neighbor nodes $i, p$, and $q$ (neighbor nodes/or potentially upstream nodes) in order to join the multicast group $m$ and received the Route-Reply messages from each node that include path costs according to their best upstream node/path to group. The received path cost as well as other attribute values are accounted in metric formula to choose the best path. For example, as shown in Figure3-4 the Join-Reply messages that j receives are $\left(Q_{m}(i), W_{m}(i)\right.$, $P I C_{m}(i)$ and $Q_{m}(p), W_{m}(p), P I C_{m}(p)$ and $Q_{m}(q), W_{m}(q), P I C_{m}(q)$ respectively from neighbors $i$, $p$, and $q$.

Then node $\mathrm{j}$ calculates its best path cost through one of its upstream nodes $\mathrm{i}, \mathrm{p}$ or $\mathrm{q}$, as:

$$
P I C(j)=\operatorname{LIC}(j)+P I C_{m}^{\text {Upstream }}
$$




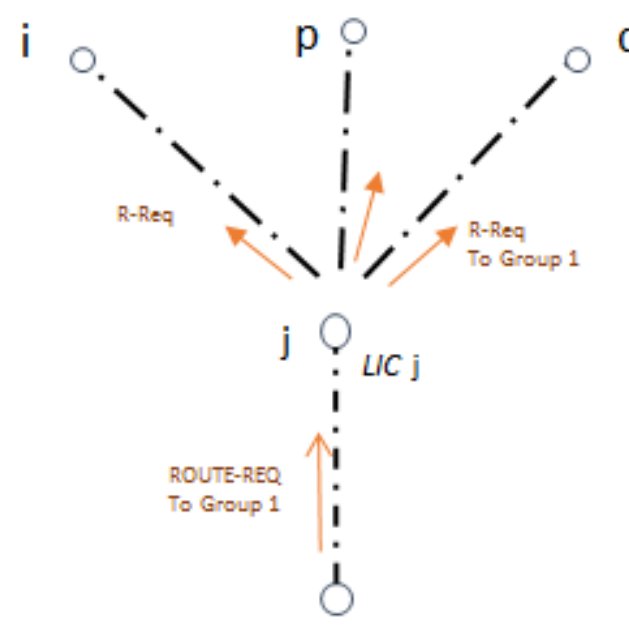

A

send ROUTE-REQUEST to group 1

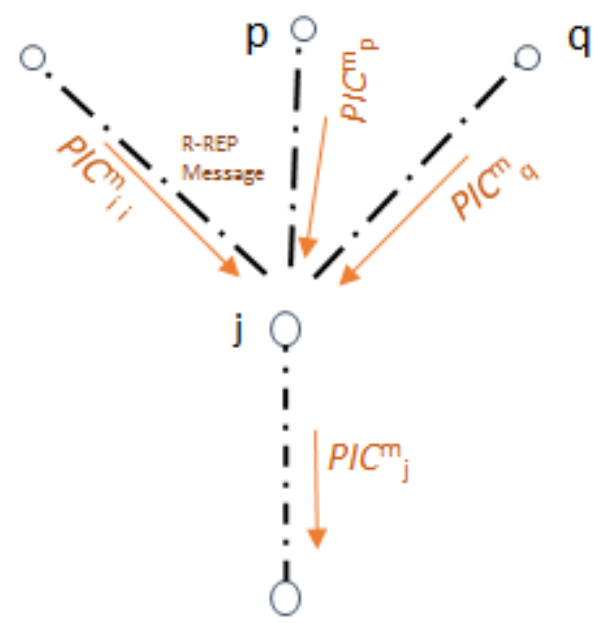

A

Route-Reply Message

Figure 3-5: IMRP Route Request and Route Reply

\subsubsection{Broadcast Benefit Factor $\left(\boldsymbol{\beta}_{\mathrm{i}}\right)$ :}

By virtue of broadcast nature of wireless medium when a node transmits a (data) packet, all its neighboring transceivers receive that packet. Hence, when a node transmits a multicast data packet to m multicast nodes, it needs only single transmission to make. This property of broadcast nature of wireless medium in multicasting can be exploited to reduce the number of transmissions and is called WMA (Wireless Multicast Advantage) [ 5]. In our design of multicast routing, we take into account in our path metric the WMA, which we call Broadcast Benefit factor of our metric $\left(\beta_{\mathrm{i}}\right)$. The overall benefit of $\beta$ is to reduce the number of forwarding nodes in a neighborhood, which in turn reduces the amount of inter-flow interference among multiple flows. The $\beta_{k}$ at node $k$ wil be $n$ If nodes $p 1, p 2, .$. and $p_{n}$ joined to node $k$..

In figure 3.5, node A requested to join to an existing multicast tree for group\#1, it is shown how we may differantiate the paths with Broadcast Benefit factors advertised from nodes $B, C$, and D. The route with higher value of $\beta$ is taking more benefit of broadcasting. As result routes adverised by node $\mathrm{C}$ need less forwarder nodes in the network comparing to the routes advertised by node $b$ and $d$. 


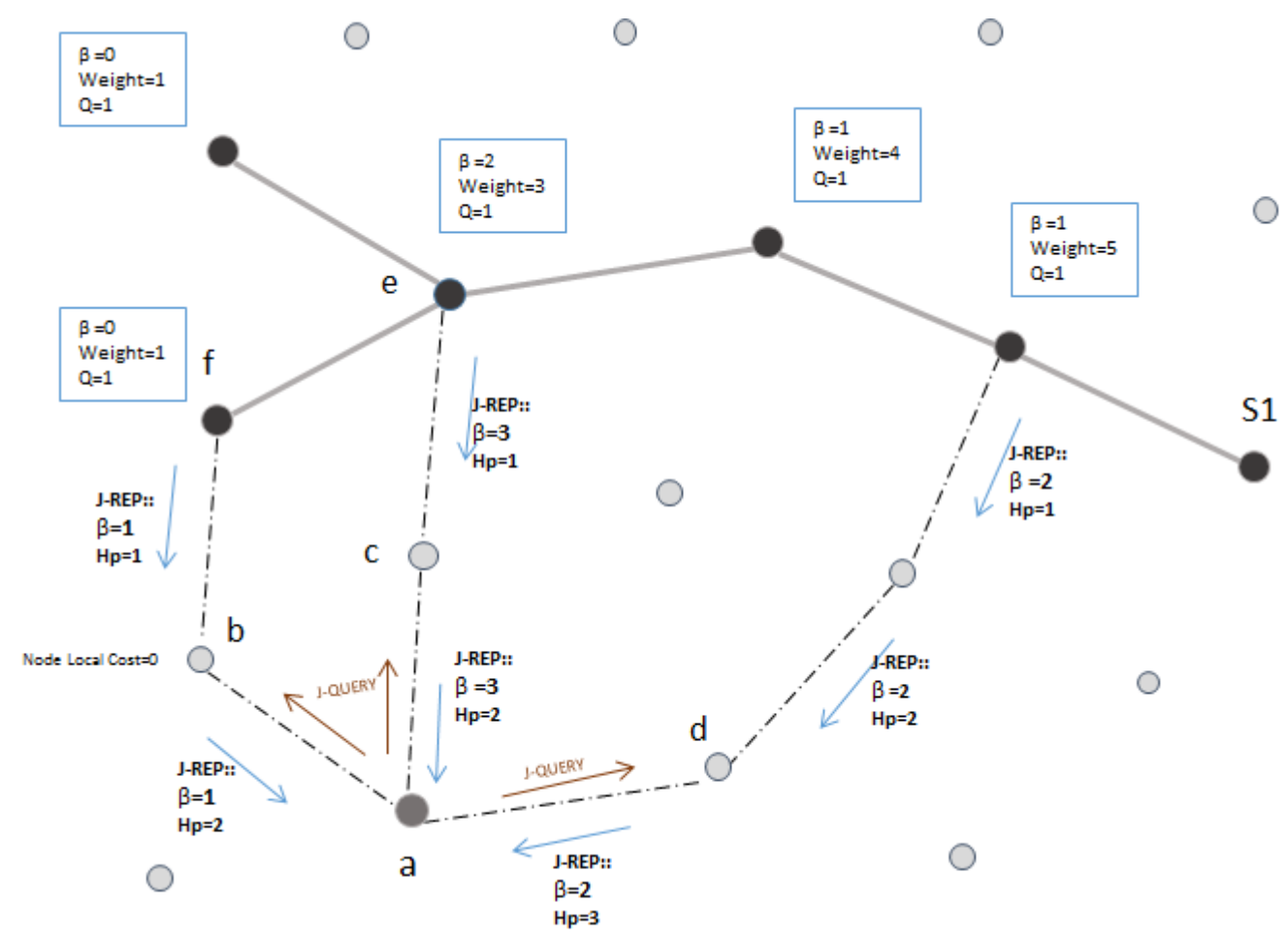

Figure 3-6 Example scenario 1: Broadcast Benefit Factor in Route Selection

\subsubsection{Hop Count Factor $(H C)$}

Hop Count $(H C)$ is still a part of our metric as an important factor. In addition to Interference Cost and Broadcast Benefit values, we also consider minumum hop count as the last part of our metric, which in turn takes the number of forwarder nodes into account alog the orther defined parameteres. 


\subsection{IMS Metric:}

Based on all prevoiusly described attributes, we can introduce our final metric IMS ( Interference-aware metric for Multicast routing over Single radio WMN). This is a composite metric that is a weighted combination of three attributes: Path Interference Cost, Broadcast Benefit and Hop Count. The weights provides relative preference in our route selection algorithm. In our composite metric formula, we multiply the three attributes by $3 \mathrm{~K}$ factors $\mathrm{k} 1$, $k 2$, and $k 3$ so that, $K 1>>K 2>>k 3$. (Our defult values: $K 1=80, k 2=10, k 3=1$ )

$\mathrm{K} 1$ is a multiple of Path Interference Cost as our first preference, $\mathrm{K} 2$ is a multiple of Broadcast Benefit Factor as second preference, and $\mathrm{K} 3$ is the multiple of the hop count as last preference.

The general equation to calculate the IMS metric is as follows:

$$
\mathrm{IMS}=\mathrm{K} 1 . \mathrm{PIC}-\mathrm{K} 2 . \beta+\mathrm{k} 3 \cdot \mathrm{HC}
$$

Adjusting the metric weights in deferent conditions of traffic demands by Network Admin can help to obtain the desired output performance. For example, in the case of increasing number of the receiver nodes, we increase $\mathrm{K} 1$ and K2, and lower K3. Because obviously we have to scarify the shortest path (minimum hop count) in the routing or end to end delay and instead increase the ratio of Packet Delivery. We do vice versa when we have less demands, the metric can be converted to Distance Vector metric like MAODV to have the better results. 


\subsection{Further Example Scenarios:}

Scenario 2:

There are two flows corresponding to multicast groups 1, and 2 respectively, shown in the network of Figure 3.7. Node $A$ wants to join to group 1 and broadcasts its Join-Request. Base on the advertised routes that $A$ receives from its neighbors $B, C$, and $D$, it calculates the lowest metric through node $D$. In turn, node $D$ computes its best potential route via $E$ then $F$ Therefor Join Confirmations are sent from A up to node F.

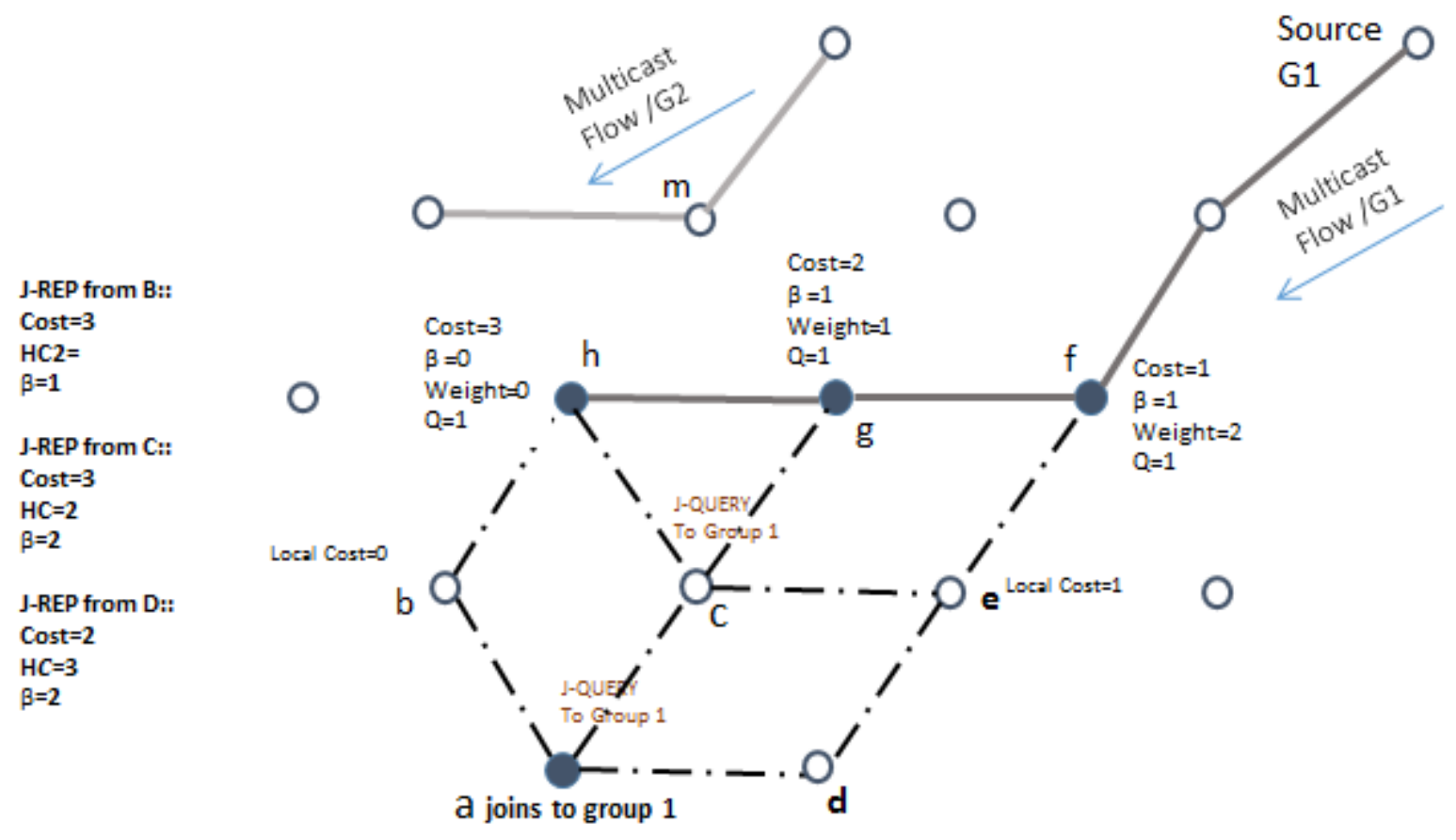

Figure 3-7 Example scenario 2

$I M S_{m j-p}=K_{1} * P I C_{m}(p)-K_{2} * \beta(p)+K_{3} *\left(H C_{m}(p)+1\right)$

b: IMS $(m 1$, via $B)=K_{1} * 3+K_{2} * 2-K_{3} * 1$

c: IMS $(\mathrm{m} 1$, via $\mathrm{C})=\mathrm{K}_{1} * 2+\mathrm{K}_{2} * 2-\mathrm{K}_{3} * 2$

d: $\operatorname{IMS}(\mathrm{m} 1$, via $\mathrm{D})=\mathrm{K}_{1} * 1+\mathrm{K}_{2} * 3-\mathrm{K}_{3} * 2$ 


\section{Scenario 3:}

In this case, we show how to incorporate the weight attribute in the proposed metric can lead choosing the best path with higher total throughput. There are two flows 1 and 2 running in the network shown in figure 3-8 belong to group 1, and 2 respectively. Node A need to join group 2. Due to location of node A, there is no other choice unless to cross the group 1's existing tree, however, here are the possible paths to join group 2:

Path1: A-C-G-M, path2: A-B-G-M, path3: A-B-H-M

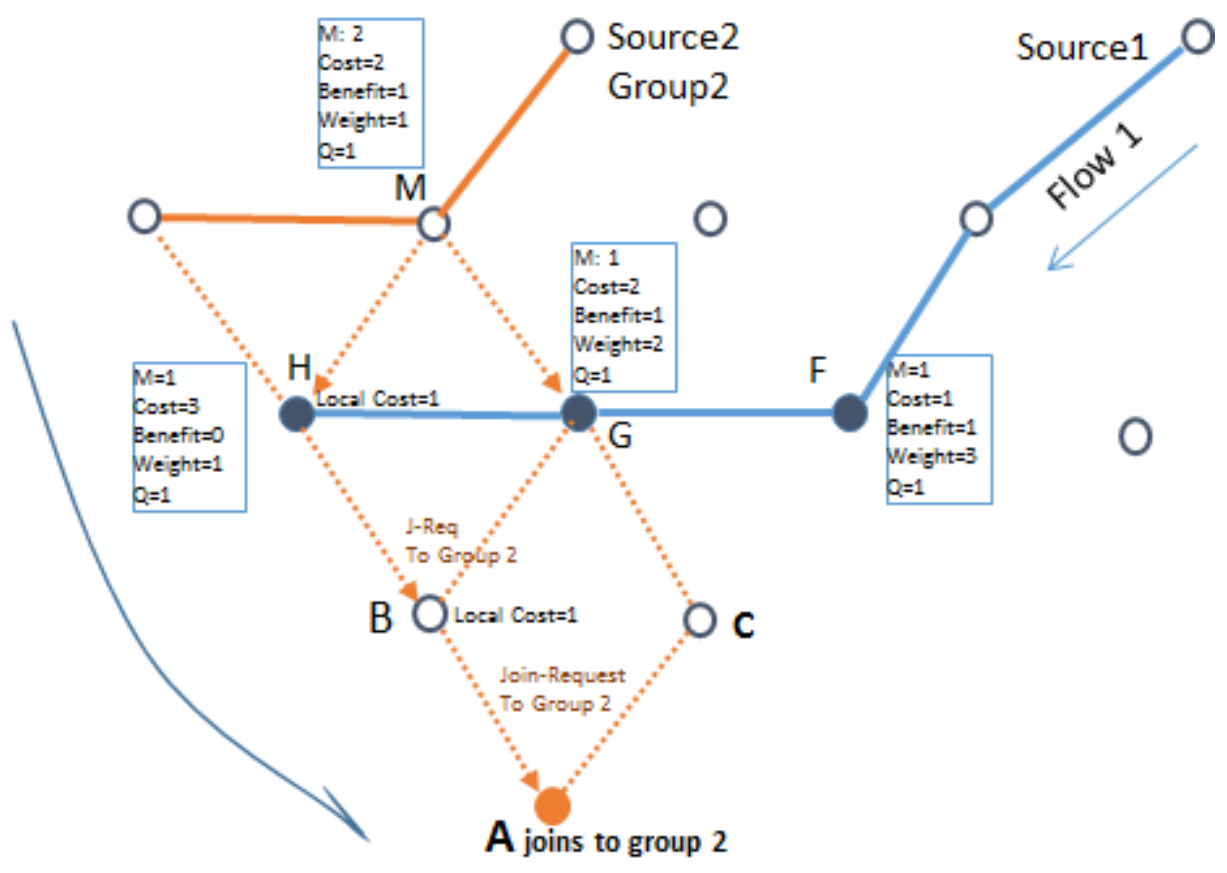

Figure 3-8 Example scenario 3

As per our metric:

$\mathrm{IMS}_{\mathrm{mAv} \text { via Up(u) }}=\mathrm{K}_{1} * P I C_{\mathrm{m} \mathrm{Up}(\mathrm{u})}-\mathrm{K} 2 * \beta_{\mathrm{Up}(\mathrm{u})}+\mathrm{K}_{3} *\left(H C_{\mathrm{m} \mathrm{Up}(\mathrm{u})}+1\right)$

$I_{\mathrm{MS}}$ (A-B-H-M) $<\mathrm{IMS}_{\mathrm{m}(\mathrm{A}-\mathrm{C}-\mathrm{G}-\mathrm{M})}<\mathrm{IMS}_{\mathrm{m} \text { (A-B-G-M })}$

Obviously, joining node $A$ to group 2 by node $\mathrm{G}$ will cause more throughput degradation on receivers $H$, and $G$ on flow 1 whereas passing by node $H$ will have less effect on receivers on flow1. However, on both paths A-B-G-M, and A-B-H-M, node A expects to have almost same performance. In next chapter, we show the results on simulated topologies and compare the results with our purposed metric. 


\section{Chapter 4}

\section{Simulation and Results}

In this chapter we evaluated our routing algorithm and metrics through simulation in a wireless mesh network with static infrastructure nodes, by multiple source/multiple group scenario. We used Riverbed Modeller 17.5 simulator. Figure 4-1 shoes the physical base topology implemented in Riverbed. We evaluated the performance of IMRP in handling multiple flows in WMN in terms of total packet delivery ratio, and total throughput. We compared the performance of IMRP with PIM-SM.

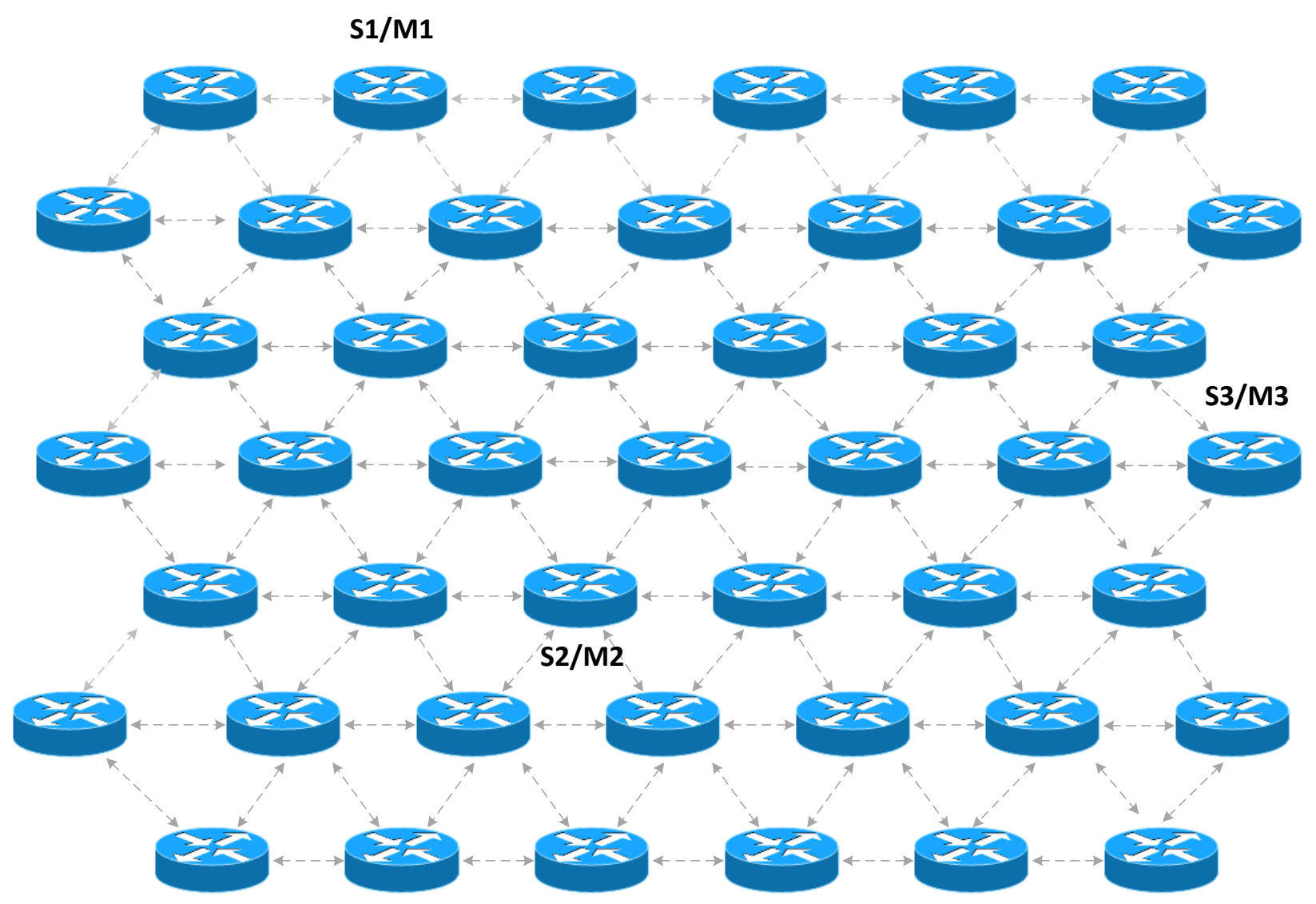

Figure 4-1 Physical base topology

In section 4.1 we introduce the base topology and discuss the network model and main assumption and corresponding configuration and setting in the model. In section 4.2 we introduce and define our performance metrics. 
In the last section, 4.3 we show the experimental results under each protocol and analyse them based on our performance metrics.

\subsection{Simulated Environment}

In this simulation, we consider a wireless mesh network of 50 infrastructure nodes which is called mesh nodes. All mesh nodes are considered stationary in our simulation. We implement 802.11 MAC in all nodes. The multicast sources generate Constant Bit Rate Multicast traffic. Also we assume Multicast traffic is high enough and it is assumed to be the main traffic so that the impact of the controlling packets are negligible.

- Network Topology: There 50 stationary mesh nodes that are placed in a $2.5 \times 2.5$ kilometers campus area. So that each node may have data connection with 6 other nodes around at equal distance around $\mathbf{3 0 0}$ meters with each other. Each mesh router serves the internal local network and workstations over wired Ethernet connections. There are few mesh routers inside WMN that are connected to gateway to outside and global network.

- Physical Layer/ MAC Layer: We fixed the transmit power for all the nodes at twenty milliwatts (0.020 watt), and also set "Packet Reception-Power Threshold" value for all of them to "-95" dbm. For the buffer size, we used $1024 \mathrm{~Kb}$ that is the Riverbed default value. Also, the nodes need to have sub-interfaces on per link. The physical layer standard is $802.11 \mathrm{~b}$ (Direct Sequence) with a data rate of $11 \mathrm{Mbps}$ is assumed for all the nodes, which can be configured overall. The access point functionality is disabled at the nodes.

- Unicast protocol: We configured OSPF in all of the nodes including receivers and routers in the network.

- Multicast protocol: We chose PIM-SM (and IGMP) as a base protocol to compare the performance of IMRP because PIM-SM is widely used multicast protocol in real networks. Rendezvous Point Node (core or RP) per group is statically selected and 
configured for all of the nodes as well as Multicast group address that they are going to join. The time when the nodes join multicast group varies per node and the hello packet interval is 30 seconds with a holding time of 105 seconds.

- Traffic:

We use average packet size 1000 Bytes (250 Words). Multicast traffic from sources starts at 300th Sec in all of our scenarios.

We need to make some minor changes in the simulation set up for each simulation scenario that we discuss at the beginning of sections 4.3. We run the simulator for 50 minutes for each scenario that results in generating traffic flow for at least 45 minutes.

\subsection{Performance Metrics}

We consider two performance metrics as defined below:

1- Throughput is computed at each receiver as the total successful multicast packet delivered in receiver nodes

2- Average packet delivery ratio (PDR) of a multicast group represents the PDR average of all receivers in the group. The PDR of a receiver is the number of data packets actually delivered to the receiver versus the number of data packets that should have been received. In our study, since we consider the total performance of all concurrent multiples flows belonging to multiple groups, our calculation is based on the following formula for Total Delivery Ratio of all groups (TGPDR):

TGPDR $=($ SP1 $*$ PDR G1 + SP2 PDR G2 +...SPn*PDR Gn) $/(L 1+L 2+\ldots+L n)$

Where SP1, SP2, .. SPn are the total number of packets sent for each group 1, 2, ... respectively. $L 1, L 2, \ldots L n$ are the average of delivered packets in group $1,2, \ldots$ and $n$.

\subsection{Simulation Setup and results}

We activated three multicast sources which are connected to different mesh nodes in different areas of the wireless mesh network. Each multicast source belongs to different multicast group. 
Multiple receivers some join each group 1, 2 and 3 . Table 4.1 shows common simulation parameters.

In our simulation, we start with two receivers in a group for both PIM-SM and IMRP. Then, we gradually increase the number of receivers to discover the cut-off point. In some cases we observed some difference in throughput beyond ten depends on location of the receiver nodes which were randomly chosen. Figure 4.2 shows that the difference in throughput between PIMSM and IMRP is higher for the case when 12 receivers are connected in a group than 6 receivers.

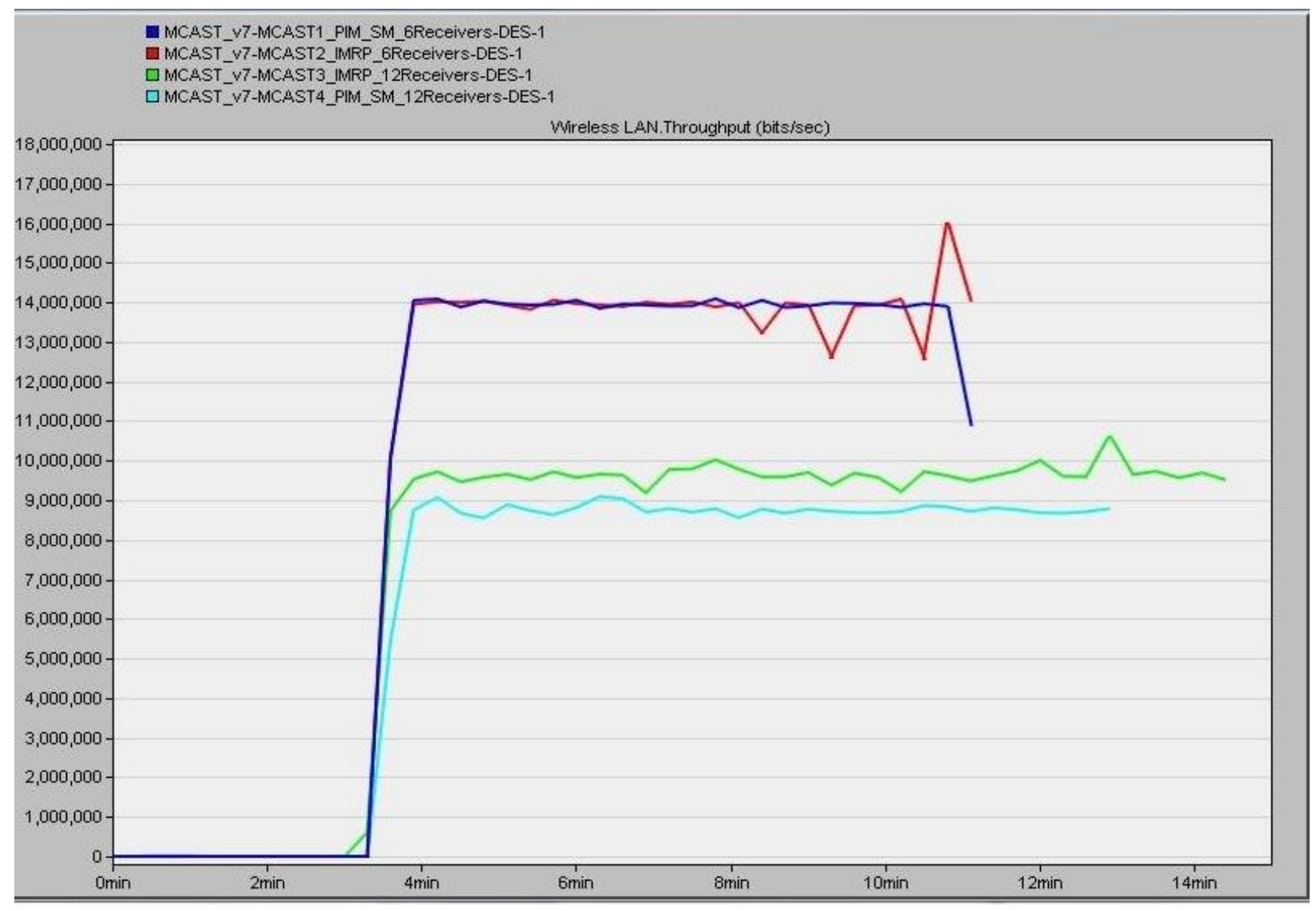

Figure 4.2 Average throughput over simulation for 6 and 12 receivers in 10 minutes For the rest of experiments we planned 8 set of simulated tests by increasing the number of receivers as $(8,12,16,20,24,28$, and 32$)$ to study the gradual change in throughput with 
increasing number of receivers. We and repeated each test 10 times by choosing random placement of receivers and taking the average of 10 runs to plot each point in Figure 4.3.

\begin{tabular}{|l|c|}
\hline Nodes in Simulation & 50 \\
\hline Physical layer model & Free space \\
\hline Physical Layer Protocol & $802.11 \mathrm{~b}$ \\
\hline MAC & CSMA/CA \\
\hline Transport Layer Protocol & UDP \\
\hline Receiver Nodes in 6 consequent run & $8,12,16,20,24,28,32$ nodes \\
\hline Hop Delay & 1.2 milliseconds \\
\hline Data Packet Size & 64 Byte \\
\hline Packet Send Delay & 30 millisecond \\
\hline Simulation Area & $2500 \times 2500$ meters \\
\hline Transmission Range & 300 meters \\
\hline Traffic Model & Constant Bit Rate \\
\hline
\end{tabular}

Table 4-1

Figure 4.3 shows the average throughput comparison for PIM-SM and IMRP. The simulation result shows negligible difference in throughput between PIM and IMRP when number of receivers connected in a group is less. 


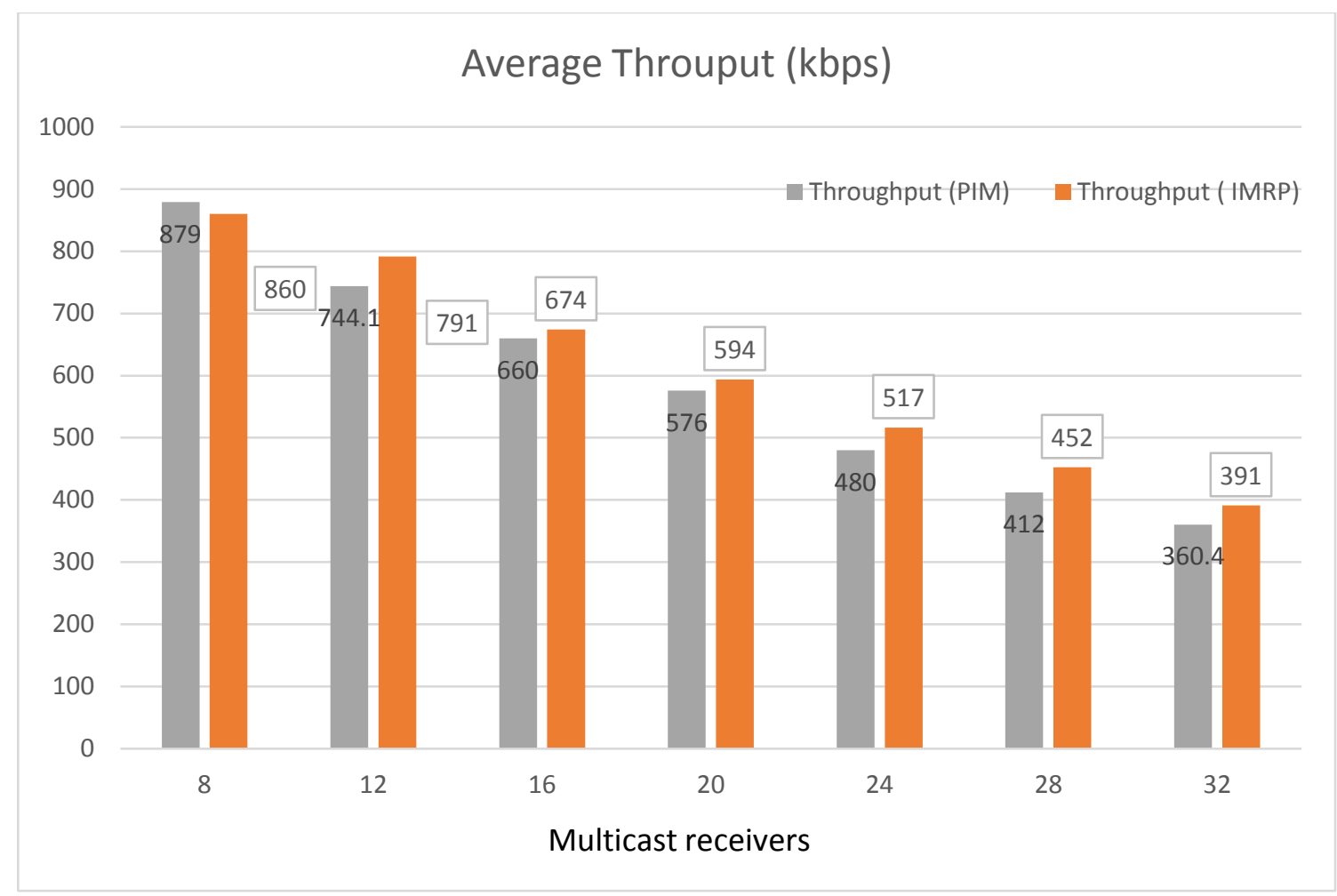

Figure 4.3: Average throughput by increasing the number of receivers

By increasing the number of receivers joining any of the three groups and consequently increasing the number of concurrent traffic paths in the network, higher interference is caused between adjacent traffic paths. , In these cases, the IMRP outperforms PIM-SM in terms of the average of throughput for all current flows in the network, which shows that IMRP improves throughput by effectively selecting paths away from higher interference. 


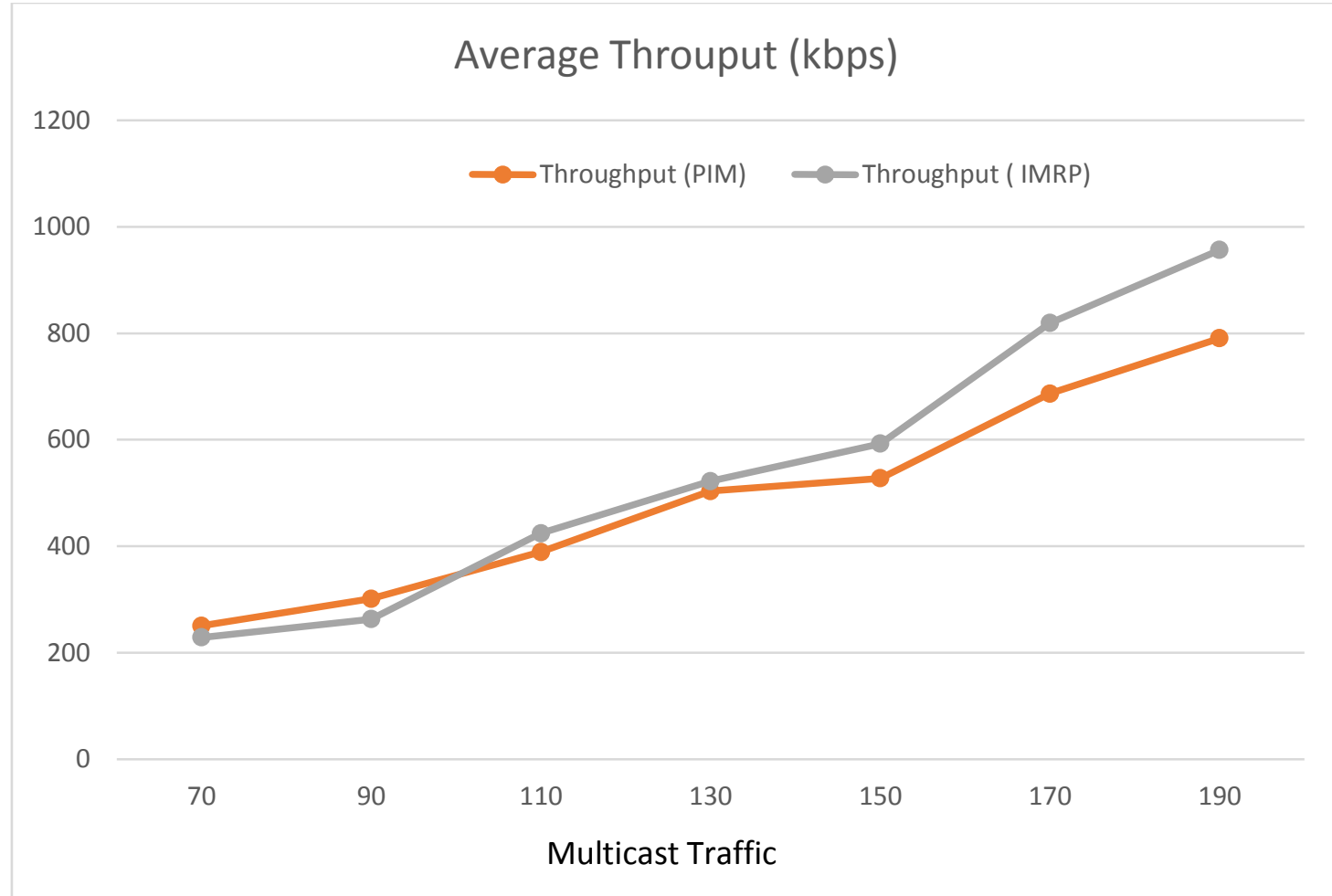

Figure 4.4 Throughput analysis as function of multicast traffic load

In the next experiment we studied the impact of multicast traffic load on the average throughput of PIM-SM and IMRP. We fixed the number of receivers in the network to 20 and increased the traffic load gradually. Higher traffic load in the network increases the interference between adjacent traffic paths and also increases the chance of congestion in shared trees serving multiple flows. The simulation results for 20 multicast receivers is shown in Figure 4.4 by increasing the traffic load from 70 packet per seconds (pps) to 190 pps. The IMRP considers interference in path selection process and routes the flows along paths with lower mutual interference and fewer hops. Hence, it consistently achieves higher throughput as compared to PIM-SM which does not consider mutual interference in its path selection process.

Our third test is to evaluate the average Packet Delivery Ratio (PDR) for both PIM-SM and IMRP. The PDR is the second important metrics for qualifying packet forwarding in multicast services. The PDR Performance with respect to number of group receivers in WMN is plotted in Figure 4.5. From the results shown in the figure, it is evident that as the number of group member increases, the PDR generally decreases for both PIM-SM and IMRP. However, the decrease in 
IMRP for higher number of groups and receivers is less than PIM because of decreasing mutual interference between multiple flows in the network. The improvement in PDR value for IMRP is approximately $13 \%$ higher than PIM.

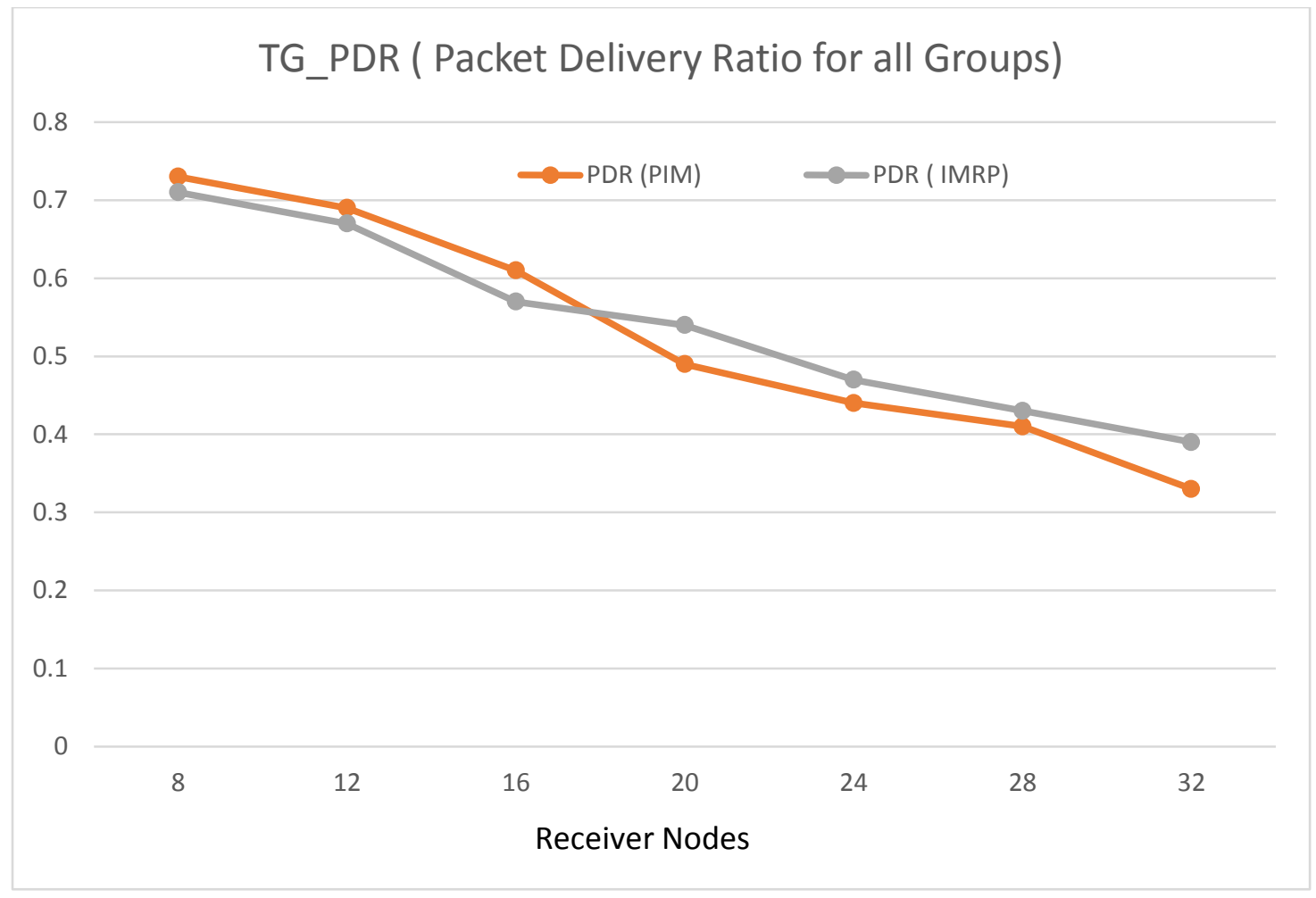

Figure 4-5: Average PDR for both PIM-SM and IMRP with increasing receivers 


\section{Chapter 5}

\section{Conclusion and future work}

\subsection{Conclusion}

In this research, we presented an interference-aware multicast routing metric scheme that comprises of two parts: a routing algorithm with necessary protocol-level details for its implementation and a routing metric. This scheme captures the impact of interference on the routing of multiple multicast flows in a WMN by modeling the amount of overall capacity of all receiver nodes in the presence of interfering nodes, and implementing the best metric for building multicast tree for each multicast group.

In summary, our research addresses the following question: given a wireless mesh network and a set of source-group traffic in the network, what is the best path selection for each group of receivers given their flow demands to maximize the overall network throughput considering both intra-flow and inter-flow interferences.

Unlike previous researches, we focus on single radio WMN with fixed nodes and demands of multiple flows corresponding to multiple groups.

Our purposed routing metric outperforms non-interference-aware routing metrics (such as PIM-SM) and other interference-aware routing metrics (like ODMRIP-WCETT) in single channel WMN environments.

We evaluated our scheme through simulation in Riverbed simulator and compared its performance with market standard PIM-SM. Our model achieves performance improvement of at least $22 \%$ compared to other studied routing metrics in the scenarios considered in this study. 
In particular, our results show that in a network of higher density of multicast receivers, our proposed scheme shows higher performance as compared to PIM-SM.

\subsection{Future works}

We identify a number of areas where our proposed scheme can be improved and extended that are discussed below:

- Our scheme can be extended for multiple channel environments.

- Since we used protocol interference model in our scheme and employed simple function of distances and ranges, instead the scheme can be modified for physical interference model by capturing the exact amount of SINR in each receiver nodes.

- In addition, we assumed equal influence of environment noise for all nodes, in physical model the value of Signal to Interference plus Noise Ratio (SINR) can be taken into account for accurate route evaluations.

- In our evaluation we assumed same rate for all multicast group flows, however our metric can be extended by taking into account the link bandwidth into flow rate. 


\section{REFERENCES}

[1] E. Alotaibi*, V. Ramamurthi, M. Batayneh, B. Mukherjee, Interference-aware routing for multi-hop Wireless Mesh Networks, in: Elsevier Computer Communications Journal, 2010. [2] De Couto DSJ (2004) High-throughput routing for multi-hop wireless networks. Ph.D. dissertation, MIT.

[3] Paul AB, Nandi S (2008) Modified Optimized Link State Routing (M-OLSR) for wireless mesh networks. In: International conference on information technology 2008 (ICIT'08).

[4] J. Padhye, S. Agarwal, V. Padmanabhan, L Qiu, A. Rao b. Zill, Estimation of link interference in static multi-hop wireless networks, in: Proceedings of IMC, 2005.

[5] M. Cagalj, P. Hubaux, C. Enz, Minimum-energy broadcast in all wireless networks: NPCompleteness and distribution. Proceedings of ACM MobiCom, September 2002.

[6] RFC4601, Fenner, B., Handley, M., Holbrook, H., and I. Kouvelas "Protocol Independent .Multicast-Sparse Mode (PIM-SM): Protocol Specification (Revised)", www.ietf.org, August 2006.

[7] A. Iyer, C. Rosenberg and A. Karnik, "What is the Right Model for Wireless Channel Interference?", Proceeding of IEEE Transactions on Wireless Communication, pp 2662 - 2671, Vol. 8, No. 5, May 2009.

[8] E. Alotaibi*, V. Ramamurthi, M. Batayneh, B. Mukherjee, Interference-aware routing for multihop Wireless Mesh Networks, in: Elsevier Computer Communications Journal, 2010. [9] Vinicius C.M. Borges, Daniel Pereira, Marilia Curado, and Edmundo Monteiro, "Routing Metric for Interference and Channel Diversity in Multi-Radio Wireless Mesh Networks", P.M. Ruiz and J.J. Garcia-Luna-Aceves (Eds.): ADHOC-NOW 2009, LNCS 5793, pp. 55-68, Springer 2009.

[11] D. Waitzman, C. Partridgeb, and S. Deering, "Distance Vector Multicast Routing Protocol (DVMRP)," Network Working Group, RFC-1075, November 1988.

[12] J. Moy, “Multicast Extensions to OSPF," RFC-1584, March 1994. 
[13] S.E. Deering, D. Estrin, D. Farinacci, V. Jacobson, C-G Liu, and L. Wei, "The PIM Architecture for Wide-Area Multicast Routing," IEEE/ACM Transactions on Networking, vol. 4, no. 2, April 1996.

[14] Elizabeth M.Royer and Charles E. Perkins, "Multicast Ad hoc OnDemand Distance Vector (MAODV) Routing", Internet Draft, draft-ietf-manet-maodv-OO.txt , July 2000.

[15] Deploying IP Multicast in the enterprise / Thomas Maufer, 2008.

[16] C.E. Perkins, E. B. Royer, S. Das, Ad hoc On-Demand Distance Vector (AODV) Routing, IETF RFC 3684 (2004).

[17] Sung-Ju Lee, Mario Gerla, and Ching-Chuan Chiang, ODMRP, On-Demand Multicast Routing Protocol.

[18] J.G. Jetcheva and David B. Johnson. Adaptive demand-driven multicast routing in multi-hop wireless ad hoc networks. In Proceedings of the ACM International Symposium on Mobile Ad Hoc Networking and Computing (MobiHoc), October 2001.

[19] V.Mallaiah et. al. / International Journal of Engineering Science and Technology Vol. 2 (10), 2010, “ MULTICAST ZONE ROUTING PROTOCOL IN WIRELESS MOBILE ADHOC NETWORKS.

[20] C-K. Toh: Guillermo Guichal, and Santithorn Bunchua “ABAM : On-Demand AssociativityBased Multicast Routing for Ad Hoc Mobile Networks", Vehicular Technology Conference, 2000. IEEE-VTS Fall VTC 2000. $52^{\text {nd }}$.

[21] Ching-Chum Chiang, Mario Gerla, Livia Zhang. Forwarding Group Multicast Protocol (FGMP) for Multihop, Mobile Wireless Networks. ACMBaltzer Journal of Cluster Computing: Special Issue on Mobile Computing, 1(2), 1998.

[22] Johnson, D.B. and D.A. Maltz, 1996. Dynamic source routing in ad hoc wireless networks. Carnegie Mellon University. 
[23] Couto, D.S.J.D., D. Aguayo, J. Bicket and R. Morris, 2003. A high-throughput path metric for multi-hop wireless routing. Proceedings of the 9th Annual International Conference on Mobile Computing and Networking, Sept. 14-19, ACM Press, San Diego, CA, USA., pp: 134-146.

[24] R. Draves, J. Padhye and B. Zill, 2004. Routing in multi-radio, multi-hop wireless mesh networks. Proceedings of the 10th Annual International Conference on Mobile Computing and Networking, Sept. 26-Oct. 01, ACM Press, Philadelphia, PA, USA., pp: 114-128.

[25] Draves, R., J. Padhye and B. Zill, 2004. Routing in multi-radio, multi-hop wireless mesh networks. Proceedings of the 10th Annual International Conference on Mobile Computing and Networking, Sept. 26-Oct. 01, ACM Press, Philadelphia, PA, USA., pp: 114-128.

[26] Subramanian, A.P., M.M. Buddhikot and S. Miller, 2006. Interference aware routing in multi-radio wireless mesh networks. Proceedings of the $2^{\text {nd }}$ IEEE Workshop on Wireless Mesh Networks, Sept. 25-28, IEEE Xplore Press, Reston, VA., pp: 55-63.

[27] C. Gui and P. Mohapatra. Effi cient overlay multicast for mobile ad hoc networks. In Proc. of IEEE WCNC, March 2003.

[28] C. Gui and P. Mohapatra. Scalable multicasting for mobile ad hoc networks. In Proc. of IEEE INFOCOM, March 2004.

[29] J. G. Jetcheva and D. B. Johnson. Adaptive Demand-Driven Multicast Routing in Multi-Hop Wireless Ad Hoc Networks. In Proc. Of ACM MobiHoc, October 2001.

[29] L. Ji and S. Corson. Differential Destination Multicast-A MANET Multicast Routing Protocol for Small Groups. In Proc. of IEEE INFOCOM, April 2001.

[30] S.-J. Lee, M. Gerla, and C.-C. Chiang. On-Demand Multicast Routing Protocol. In Proc. of IEEE WCNC, September 1999.

[31] J. Luo, P. T. Eugster, and J.-P. Hubaux. Route Driven Gossip: Probabilistic Reliable Multicast in Ad Hoc Networks. In Proc. of IEEE INFOCOM, March 2003.

[32] E. M. Royer and C. E. Perkins. Multicast operation of the ad-hoc on-demand distance vector routing protocol. In Proc. of MobiCom, August 1999.

[33] P. Sinha, R. Sivakumar, and V. Bharghavan. MCEDAR: Multicast Core-Extraction Distributed Ad hoc Routing. In Proc. of IEEE WCNC, September 1999. 
[33] Sabyasachi Roy, Dimitrios Koutsonikolas, Saumitra Das, and Y. Charlie Hu "High-Throughput Multicast Routing Metrics in Wireless Mesh Networks" Proceedings of the 26th IEEE International Conference on Distributed Computing Systems (ICDCS'06) 0-7695-2540-7/06 @ 2006.

[34] ITU-T Rec. H.264 (ISO/IEC 14496-10). Advanced Video Coding for Generic Audiovisual Services (April 2013).

[35] Internet Draft Protocol Independent Multicast - Sparse Mode (PIM-SM): Protocol Specification (Revised), Internet Engineering Task Force, PIM WG, 1 March 2002.

[36] Internet Draft Protocol Independent Multicast - Dense Mode (PIM-DM): Protocol Specification (Revised), Internet Engineering Task Force, PIM WG, 15 February 2002 (draft-ietf-pim-dm-new-v2-01).

[37] Effects of Interference on Wireless Mesh Networks: Pathologies and a Preliminary Solution Yi Li, Lili Qiu, Yin Zhang, Ratul Mahajan, Zifei Zhong, Gaurav Deshpande, and Eric Rozner, November 2007.

[38] Modeling Interference in Wireless Ad Hoc Networks, Paulo Cardieri, IEEE Communication SURVEYS VOL. 12, NO. 4, FOURTH QUARTER 2010. 